\title{
Robust constrained stabilization of boost DC-DC converters through bifurcation analysis
}

\author{
C. Yfoulis ${ }^{1}$, D. Giaouris ${ }^{2}$, F. Stergiopoulos ${ }^{1}$, C. Ziogou ${ }^{2}$, S. Voutetakis ${ }^{2}$ and S. Papadopoulou ${ }^{1,2}$ \\ ${ }^{1}$ Automation Engineering Department, Alexander Technological Educational Institute of Thessaloniki, 57400, Thessaloniki, P.O.Box 141, GREECE \\ ${ }^{2}$ Chemical Process Engineering Research Institute (C.P.E.R.I.), Centre for Research and Technology Hellas (CE.R.T.H.), 6th km Harilaou-Thermis, GR-57001, \\ Thermi, Thessaloniki, GREECE
}

\begin{abstract}
This paper proposes a new methodology for designing robust affine state-feedback control laws, so that wide-range safe and efficient operation of switched-mode DC-DC boost converters is guaranteed. Several undesirable nonlinear phenomena such as unstable attractors and subharmonic oscillations are avoided through bifurcation analysis based on the bilinear averaged model of the converter. The control design procedure also relies on constrained stabilization principles and the generation of safety domains using piecewise linear Lyapunov functions, so that robustness to supply voltage and output load variations is ensured, while input saturation is avoided and additional state constraints are also respected. The technique has been numerically and experimentally validated.
\end{abstract}

Keywords: DC-DC boost converter, bifurcation analysis, constrained stabilization, piecewise-linear Lyapunov functions

\section{Introduction}

The control of switched mode DC-DC converters has attracted considerable interest in recent years from the control community, due to the great practical importance and the widespread use of the DC-DC conversion technology. It is an interesting control problem with special characteristics and challenges, such as hard state and control constraints, the need for a fast and accurate static and dynamic performance, robustness to unpredictable but bounded supply voltage and load variations, low complexity of implementation and corresponding low-cost hardware. The basic challenge is that switched-mode converters are highly nonlinear systems $[1,2,3]$ hybrid in nature, since they involve high-frequency switching among two different modes of operation.

The main control objective of such converters is output voltage regulation in the presence of load and supply voltage variations. PID controllers are designed on the basis of a linearized model while other nonlinear, robust and optimal control methodologies consider more complex mathematical model representations of the hybrid dynamics which lead to more efficient controllers, at the expense of an increased complexity. Many advanced control techniques have been applied recently to this problem, e.g. $[4,5,6,7]$. In particular, model predictive control (MPC) techniques offer optimal solutions subject to all state and control constraints. Unfortunately, the price that has to be paid is the generation of highly complex piecewise affine control laws and corresponding partitions of the state-space, which give frequently rise to additional undesired effects, such as chattering (high frequency switching) between neighboring regions

\footnotetext{
This paper was not presented at any IFAC meeting. Corresponding author: C. Yfoulis, cyfoulis@autom.teithe.gr, Fax : +302310498390 .

你吱 This work is implemented through the Operational Program "Education and Lifelong Learning", co-financed by the European Union (European Social Fund) and Greek national funds, program "Archimedes III".
}

[8]. Furthermore, as explained in [9] and other relevant works, when resorting to sub-optimal and/or approximate solutions a posteriori stability checks and multiple trials may be necessary, where it might be unclear how the MPC setup is to be modified for a solution to be found.

A nonlinear-system approach using piecewise-linear differential inclusions, piecewise-quadratic Lyapunov functions and Linear Matrix Inequalities (LMIs) convex optimization has been employed in [10] for the same problem. Moreover, in [11, 12] parameter-dependent Lyapunov functions and corresponding time-varying parameter-dependent (gain-scheduled) control laws are proposed.

Furthermore, set-theoretic approaches to the constrained stabilization of power converters on the basis of bilinear dynamics have been recently proposed, see $[13,9,6,14]$ and references therein. In these works linear static state-feedback controllers can be found by generating polytopic contractive sets induced by corresponding piecewise-linear (polyhedral) Lyapunov functions while taking into account both state and input constraints.

All these publications propose various novel control strategies that improve the converter's controlled behavior but, to the best of the authors knowledge, they do not address the issue of multiple attractors. For a special form of state-feedback controllers (the so-called Lyapunov-based control) the generation of multiple equilibria using the averaged converter model has been recently studied in [15]. It has been shown that bifurcation phenomena are completely avoided in the absence of controller mismatch, but they appear in the case of a mismatch between the values used by the controller and the real system dynamics. However, as demonstrated recently in [16], for a general statefeedback controlled boost converter, it is possible to have more complicated bifurcation phenomena. Therefore, it is necessary to carefully investigate these instabilities (that can greatly reduce the converter's lifetime) and to propose a complete con- 
troller design methodology that enhances the performance of the converter (over conventional methods) while at the same time avoiding unwanted nonlinear phenomena such as subharmonic oscillations, multiple attractors and chaos.

To this end, a distinguishing feature of the approach described in this paper is the investigation of the effect of varying parameters (supply voltage and output load), and state-feedback gains on the generation of undesirable nonlinear phenomena, due to the switching action. Bifurcation phenomena that can give rise to multiple equilibria and unstable attractors, as well as subharmonic oscillations are detected using bifurcation analysis. The material presented in $[14,16]$ is extended by fully investigating the aforementioned nonlinear phenomena for all possible variations of the uncertain parameters. Relating the presence of such phenomena with the range of parameters and the controller gains is important, and must be taken into account during the design procedure.

Furthermore, another novelty presented in this work is the extension of the main stabilization mechanism to robust tracking, in order to ensure robust stability and performance over a wide range of operating conditions, including special treatment of the performance during startup without imposing extra rate constraints. The use of recently proposed flexible piecewise linear Lyapunov functions and corresponding efficient raygridding iterative algorithms [17] allows the generation of nearmaximal attraction domains, while taking into account state constraints and saturation nonlinearities. Therefore, the main contribution of this work is the development of a systematic design procedure based on the combination of constrained stabilization principles and bifurcation theory.

This paper is organized as follows. In section 2, the boost converter model is described and a motivating example is presented. A complete analysis and prediction of bifurcation phenomena on the basis of the averaged bilinear converter dynamics, is next described in section 3 . In section 4 the new bifurcation based criteria are combined with further requirements and specifications in a constrained stabilization setting to form a novel and complete design procedure, which is successfully implemented in an illustrative example in section 5. The final section concludes by summarizing our main conjectures and discussing key issues and future work.

Notation : In this paper, $\mathbb{R}$ denotes the real numbers and $\mathbb{R}^{n}$ is the vector space of $\mathrm{n}$-dimensional real vectors. Boldface upper case letters denote matrices, while boldface lower case letters are used for vectors. All vectors are assumed to be column vectors. $\mathrm{x}^{T}$ denotes the transpose and $x_{i}$ the $\mathrm{i}$-th component of vector $\mathbf{x}$. If $P$ is a set in $\mathbb{R}^{n}, r i\{P\}$ and $\partial P$ denote the relative interior and the boundary of $P$, respectively.

\section{Preliminaries and a motivating example}

\subsection{The boost converter}

A typical step-up (boost) DC-DC converter topology as in Fig. 1 with fixed frequency switching between two linear circuits is considered. It is designed to provide a higher DC output voltage at some desired reference level $V_{\text {ref }}>V_{i n}$, where $V_{i n}$ is the DC supply voltage in the input. There are two state variables, the capacitor voltage $v_{C}$ and the inductor current $i_{L}$, and if the state vector is defined as $\mathbf{x}:=\left[v_{C}, i_{L}\right]^{T}$ then the system dynamics can be expressed in a continuous-time piecewise smooth form

$$
\dot{\mathbf{x}}= \begin{cases}\mathbf{A}_{o n} \mathbf{x}+\mathbf{B}_{o n} V_{i n} & , \mathrm{~S}=\text { on } \\ \mathbf{A}_{\text {off } f} x+\mathbf{B}_{\text {off }} V_{i n} & , \mathrm{~S}=\text { off }\end{cases}
$$

where

$$
\begin{gathered}
\mathbf{A}_{o n}=\left[\begin{array}{cc}
-\frac{1}{R C} & 0 \\
0 & 0
\end{array}\right], \mathbf{A}_{o f f}=\left[\begin{array}{cc}
-\frac{1}{R C} & \frac{1}{C} \\
-\frac{1}{L} & 0
\end{array}\right] \\
\mathbf{B}_{o n}=\mathbf{B}_{o f f}=\left[\begin{array}{l}
0 \\
\frac{1}{L}
\end{array}\right]
\end{gathered}
$$

and $R$ is the load resistance, $C$ the filter capacitance, $L$ the circuit inductance and $\mathrm{S}$ is the switch. The proportion of time that the switch $\mathrm{S}$ is On is called duty cycle $(d(t))$ and it can be proved that in the boost converter it relates the input and output voltages through the relation $d_{s s}=1-\frac{V_{i n}}{V_{s s}}$, where $d_{s s}, V_{s s}$ are the corresponding steady state values.

\subsection{The bilinear averaged model}

We consider the average state-space model

$$
\dot{\mathbf{x}}=\mathbf{A} \mathbf{x}+\mathbf{B} V_{i n}
$$

which involves the duty cycle $d(t) \in[0,1]$ and is the result of taking the average of the state and input matrices

$$
\begin{gathered}
\mathbf{A}=\mathbf{A}_{o n} d+\mathbf{A}_{o f f}(1-d)=\left[\begin{array}{cc}
-\frac{1}{R C} & \frac{1}{C}(1-d) \\
-\frac{1}{L}(1-d) & 0
\end{array}\right] \\
\mathbf{B}=\mathbf{B}_{o n} d+\mathbf{B}_{o f f}(1-d)=\left[\begin{array}{c}
0 \\
\frac{1}{L}
\end{array}\right]
\end{gathered}
$$

Since our control input is the duty cycle, i.e. $u(t)=d(t)$, the linearized system equations may be reformulated as

$$
\dot{\mathbf{x}}=\mathbf{A}_{1} \mathbf{x}+\mathbf{A}_{2} \mathbf{x} u+\mathbf{b}
$$

where

$$
\mathbf{A}_{1}=\mathbf{A}_{o f f}, \mathbf{A}_{2}=\mathbf{A}_{o n}-\mathbf{A}_{o f f}, \mathbf{b}=\mathbf{B} V_{i n}
$$

These equations suggest that the average converter dynamics are in a bilinear form with a non-zero equilibrium state. Simple calculations reveal that the steady-state operating conditions $\mathbf{x}_{s s}=\left[V_{s s}, I_{s s}\right]^{T}, u_{s s}=d_{s s}$ are parameter-dependent

$$
V_{s s}=\frac{V_{i n}}{1-u_{s s}}, I_{s s}=\frac{V_{i n}}{R\left(1-u_{s s}\right)^{2}}=\frac{V_{s s}^{2}}{R V_{i n}}
$$

and define the feasible equilibria region (FER) for all parameter values as

$$
\mathcal{F}=\left\{\mathbf{x} \in \mathbb{R}^{2}: \mathbf{x}=\left[V_{s s}, I_{s s}\right]^{T}\right\}
$$


The system operates in the presence of uncertainties which are the unpredictable load and the input voltage variations. Although the random variations are unknown, we consider that approximate bounds can be a priori specified

$$
V_{i n} \in\left[V_{i n}^{-}, V_{i n}^{+}\right], R \in\left[R^{-}, R^{+}\right]
$$

In this paper, we study affine state-feedback control laws

$$
u=\mathbf{k}^{T}\left(\mathbf{x}-\mathbf{x}_{s s}\right)+u_{s s}, \mathbf{k}=\left[k_{1}, k_{2}\right]^{T}
$$

guaranteeing convergence to a desired equilibrium state $\mathbf{x}_{s s}$, where $\mathbf{x}_{s s}, u_{s s}$ are given by (9), hence an integral action is not required. A detailed analysis of this control law is given in section 3 .

\subsection{A motivating example}

As a motivating example, a boost converter as in Fig. 1 is considered with nominal parameter values $L=1.5 \mathrm{mH}, C=$ $10 \mu \mathrm{F}, R=40 \Omega, V_{\text {in }}=5 \mathrm{~V}, V_{\text {ref }}=10 \mathrm{~V}$. It is assumed to work in a wide range of operating conditions, i.e. large intervals for the uncertain parameters $V_{i n} \in[3.5,6.5] \mathrm{V}, R \in[20,80] \Omega$ are considered. The typical saturation avoidance condition for the duty cycle $d \in[0,1]$ and hard safety constraints for the inductor current $0<i_{L} \leq 1.5 \mathrm{~A}$ and the capacitor voltage $0 \leq v_{C} \leq 30 \mathrm{~V}$ are imposed. The lower bound constraint $i_{L}>0$ guarantees that the converter operates in continuousconduction mode (CCM) ${ }^{1}$.

It is not difficult to use many different methodologies [14, 6] to design state-feedback laws as in (12) for nominal operating conditions, while extra state and control constraints are satisfied. However, when the converter operates in a wide operating region, several undesirable phenomena may occur. These are shown below with simulation results obtained from SIMULINK, where the exact switched model of the converter is used in a digital implementation of (12) as follows :

$$
u(n T)=d(n T)=\mathbf{k}^{T}\left(\mathbf{x}(n T)-\mathbf{x}_{s s}\right)+d_{s s}
$$

Let us consider two different state-feedback designs with similar gains (the exact design procedure is in detail presented in sections 4 and 5):

$$
\mathbf{k}_{1}=[0.043,-0.2825]^{T}, \mathbf{k}_{2}=[0.0443,-0.2324]^{T}
$$

In nominal operating conditions, both gains result in satisfactory transient responses, as seen in Fig. 2, and it can be shown that a stable (period 1) output voltage response is obtained from any initial condition. This is demonstrated in Fig. 2(a) during startup (the two responses are practically indistinguishable), and in Fig. 2(b) from a diverse operating condition (corresponding to $V_{\text {ref }}=10 \mathrm{~V}, V_{\text {in }}=3.5 \mathrm{~V}, R=20 \Omega$ ). Unfortunately, when the converter's uncertain parameters are allowed

\footnotetext{
${ }^{1}$ Although operation in discontinuous-conduction (DCM) mode is not usually undesirable, this assumption is included in order to demonstrate the possibility of including several different types of constraints in constrained stabilization design.
}

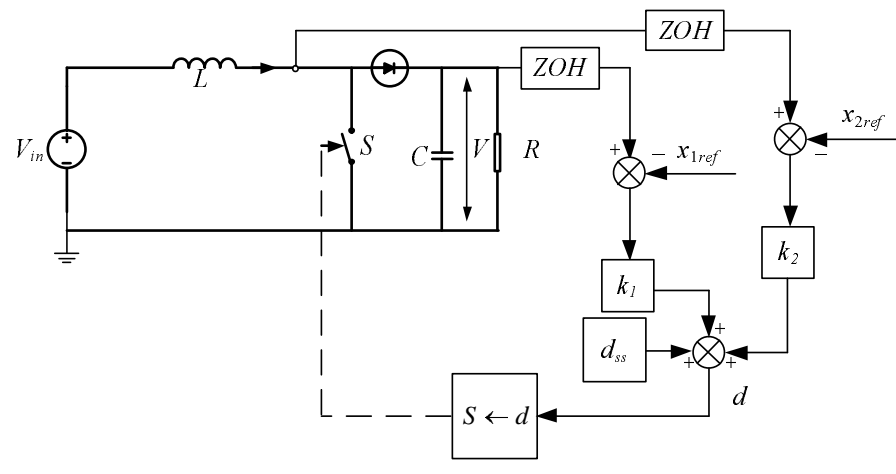

Figure 1: State-feedback controlled boost converter.

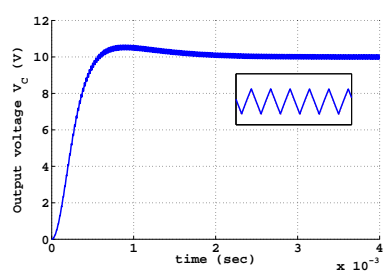

(a)

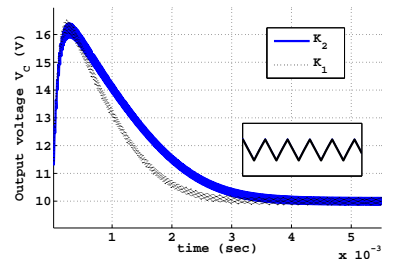

(b)
Figure 2: Transient responses with different gains $\mathbf{k}_{1}, \mathbf{k}_{2}$ are compared under nominal operating conditions $V_{\text {ref }}=10 \mathrm{~V}, V_{i n}=5 \mathrm{~V}, f_{s}=50 \mathrm{kHz}$ $R=40 \Omega$. (a) Startup transient , (b) transient from an extreme operating point $\mathbf{x}(0)=\left[\begin{array}{ll}10 & 1.42\end{array}\right]^{T}$ corresponding to $V_{\text {ref }}=10 \mathrm{~V}, V_{\text {in }}=3.5 \mathrm{~V}, R=20 \Omega$.

to deviate significantly from nominal values, several undesirable phenomena may occur, depending on the state-feedback gains. These include generation of unstable attractors and subharmonic oscillations. This is clearly depicted with the input voltage and load transient responses shown in Fig. 3, where the performance of the state-feedback controllers is stress tested by considering far from nominal operating conditions and an initial condition corresponding to a large disturbance (inside the specified parameter range). Two different switching frequencies are also tested.

The first gain $\mathbf{k}_{1}$ provides acceptable and stable period 1 responses in all cases, to a desired output voltage of $10 \mathrm{~V}$. Unfortunately, the second gain $\mathbf{k}_{2}$ fails to provide an acceptable response for the whole operating regime. In Figs. 3(a),(b) the existence of an unstable attractor drives the output voltage to a distant operating point at $67.61 \mathrm{~V}$ and $23.65 \mathrm{~V}$, respectively. In Fig. 3(c), apart from attracted to a distant equilibrium, the response is also distorted by subharmonics when a lower switching frequency $f_{s}=20 \mathrm{kHz}$ is used.

The situation can become much worse for higher state-feedback gains. For instance, let us consider a third gain

$$
\mathbf{k}_{3}=[0.06,-0.19]^{T}
$$

selected to provide an excellent initial startup response for nominal operating conditions, as in Fig. 4(a). Unfortunately, the system's response is now very sensitive to disturbances, as can be seen in Fig. 4(b). Starting from nominal operating conditions, a relatively small change of the output load from $40 \Omega$ to $50.5 \Omega$ gives rise to unstable attractors and subharmonic oscil- 

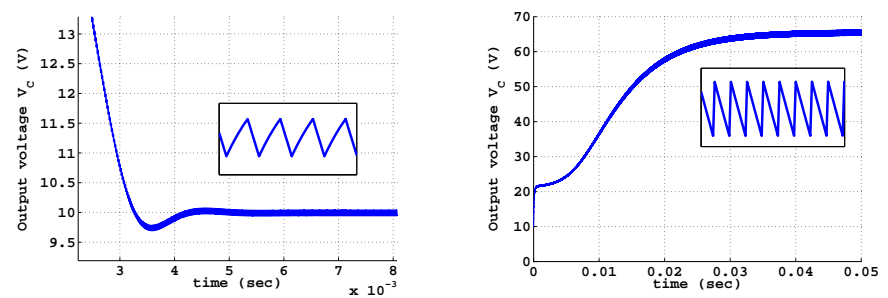

(a)
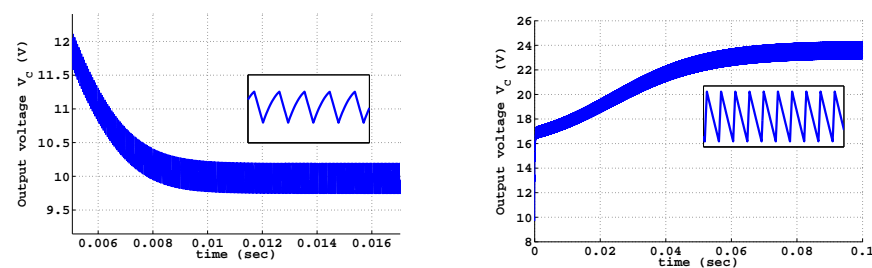

(b)

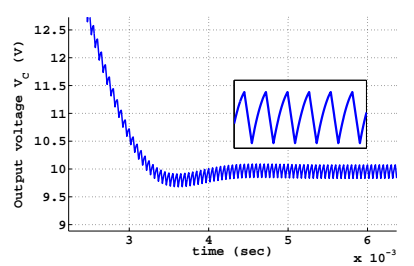

(c)

Figure 3: Output voltage responses $v_{C}(\mathrm{~V})$ vs. time $t(\mathrm{sec})$ for $V_{\text {ref }}=10$ $\mathrm{V}$ from $\mathbf{x}(0)=\left[\begin{array}{ll}10 & 1.42\end{array}\right]^{T}$ are shown for two different gains $\mathbf{k}_{1}$ (left) and $\mathbf{k}_{2}$ (right) and different operating conditions : (a) $V_{\text {in }}=6.5 \mathrm{~V}, R=77 \Omega$, and $f_{s}=50 \mathrm{kHz}$. (b) $V_{i n}=3.5 \mathrm{~V}, R=71.5 \Omega$, and $f_{s}=20 \mathrm{kHz}$. (c) $V_{\text {in }}=6.5 \mathrm{~V}, R=71.5 \Omega$, and $f_{s}=20 \mathrm{kHz}$

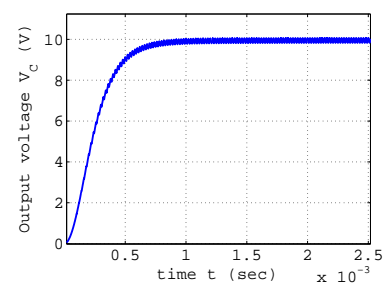

(a)

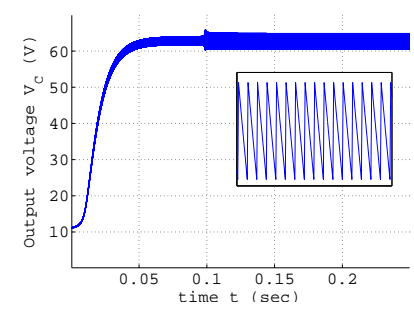

(b)

Figure 4: Output voltage responses $v_{C}(\mathrm{~V})$ vs. time $t(\mathrm{sec})$. Transient responses from $\mathbf{x}(0)=\left[\begin{array}{ll}10 & 0.5\end{array}\right]^{T}$ are shown for gain $\mathbf{k}_{3}=[0.06,-0.19]^{T}, V_{\text {ref }}=10$ $\mathrm{V}, V_{i n}=5, f_{s}=50 \mathrm{kHz}$ (a) for an initial startup for $R=40 \Omega$ (nominal). (b) for a load change from $R=40 \Omega$ to $R=50.5 \Omega$.

lations. As opposed to gains $\mathbf{k}_{1}, \mathbf{k}_{2}$ where the same phenomena are observed far from nominal operating conditions, for gain $\mathbf{k}_{3}$ the same phenomena are observed close to the nominal operating conditions, and for a sufficiently high $f_{s}=50 \mathrm{kHz}$ switching frequency, i.e. regardless of $f_{s}$.

A global perspective of the instabilities that can be observed in such converters is shown in Fig. 5, where the bifurcation diagrams were created using the exact switched model of the system. From these bifurcation diagrams it can be seen that the system goes through a saddle-node bifurcation which creates another undesired equilibrium point. Furthermore, in the case of using a sampling frequency of $10 \mathrm{kHz}$, this new equi-

librium point goes through a period doubling bifurcation that creates extra subharmonics and high current ripple ${ }^{2}$. Obviously operating in such region will greatly reduce the lifetime of the converter and therefore the proper design of the converter and the state-feedback compensator play a significant role. More specifically, the presence of a number of undesirable phenomena, arising for slightly different feedback gains, suggests the existence of critical bifurcation points (in the gain's or the uncertain parameter's space) separating safe from unsafe regions. Techniques for locating the safe regions need to be incorporated in the control design process so that the corresponding undesirable phenomena may be predicted and ruled out. Then the existence of a single feedback gain guaranteeing a stable period 1 operation and the satisfaction of all state and control constrains for an a-priori known operating range can be investigated. If such feedback gains exist, a procedure for making an optimal selection, e.g. in terms of performance must be sought.

The approach proposed in this paper deals with all aforementioned issues by resorting to a systematic and transparent design procedure complemented by useful bifurcation analysis on the basis of the continuous-time nonlinear averaged model of the converter.

\section{Bifurcation Analysis}

The results presented in the previous section suggest that the saddle-node bifurcation always pre-exists the period doubling bifurcation regardless of the converter's parameters or controller gains. This implies that it may be possible to study this behaviour of the converter using the so-called nonlinear "averaged model" . However, this model cannot fully unfold the complete dynamics of the system, as a) it ignores the fast scale phenomena induced by the switching [2] and b) in some converters (like the buck converter) the averaged model cannot even locate the existence of a saddle node bifurcation. Therefore it is imperative to validate the averaged model prior to any usage for the analysis and design of suitable control laws. While, in this case study (a boost converter) the averaged model can predict the occurrence of the saddle node, this information is worthless if a period doubling has taken place first. To this end, the authors in [16] have used the saltation matrix to study the switching effect and hence the overall monodromy matrix for the nonsmooth orbit was determined. This allowed a thorough investigation of the bifurcation phenomena and it was numerically determined that the saddle node bifurcation indeed appears first for a wide range of operating conditions. More specifically, as seen in Fig. 6 , for $f_{s}=50 \mathrm{kHz}$ the absence of any period-doubling phenomena inside the admissible range is confirmed. However, for a low frequency $f_{s}=10 \mathrm{kHz}$ such period-doubling bifurcation phenomena are clearly observed. Nevertheless, all experiments suggest that they always follow

\footnotetext{
${ }^{2}$ It has to be mentioned at this point, that further interesting phenomena such as Hopf and border collision bifurcations are observed after this period doubling instability. However, their study is outside the scope of this paper as our focus is to avoid the first 2 bifurcations (saddle node and period doubling) that will greatly deteriorate the performance of the converter.
} 


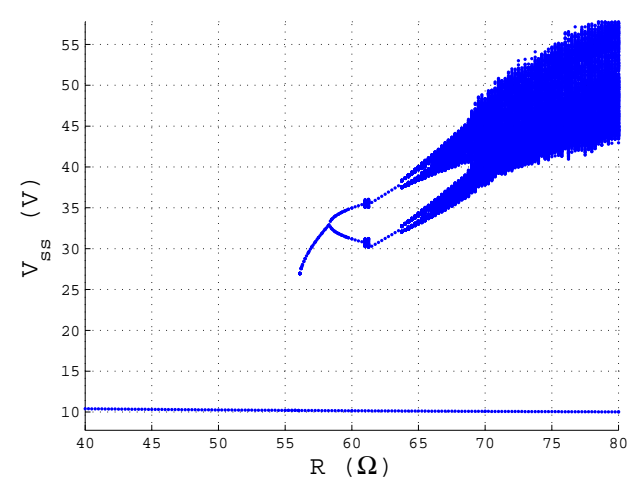

(a)

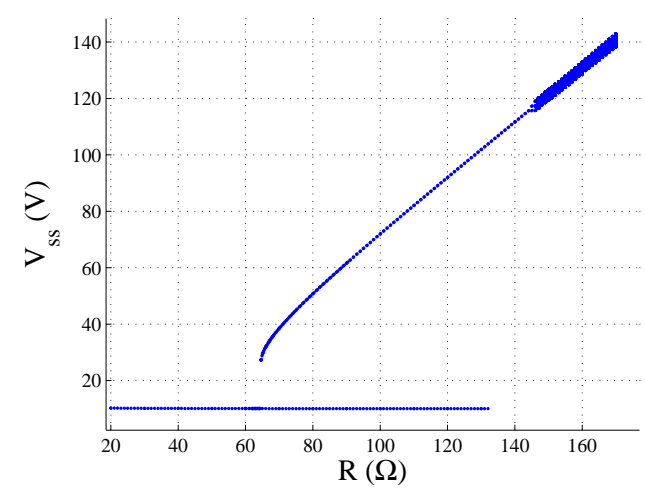

(b)

Figure 5: Brute-force bifurcation diagrams for varying load $R$, with compensating controller action, gain $\mathbf{k}_{2}=[0.0443,-0.2324]^{T}$ and (a) $f_{s}=10 \mathrm{kHz}$, (b) $f_{s}=50 \mathrm{kHz}$.

the saddle-node bifurcation points, i.e. they occur for higher deviations of the bifurcation parameters $V_{i n}, R$ from their nominal values. Therefore, it is possible to use the averaged model of the converter in order to have simpler expressions that can be fruitfully utilised in the controller design. However, the aforementioned analysis is necessary as it validates and defines operational limits of the averaged model approach presented in the following sections especially when the switching frequency is not sufficiently high.

\subsection{Parameter variation bifurcation analysis using the aver- aged model}

In this section we use the continuous-time nonlinear averaged converter's model in order to show that the appearance of multiple equilibria can be accurately predicted ${ }^{3}$. The bifurcation analysis proposed is an important complementary tool that can guide the control design procedure, as shown in the following sections.

In [15], the authors have considered a special form of the state-feedback controller (12) -the so-called Lyapunov-based

\footnotetext{
${ }^{3}$ At this point it has to be mentioned that the effect of the digital implementation ( $\mathrm{S} / \mathrm{H}$ operation) can be ignored due to high sampling rate compared to the system's bandwidth. This is also discussed in detail in section 5 .
}

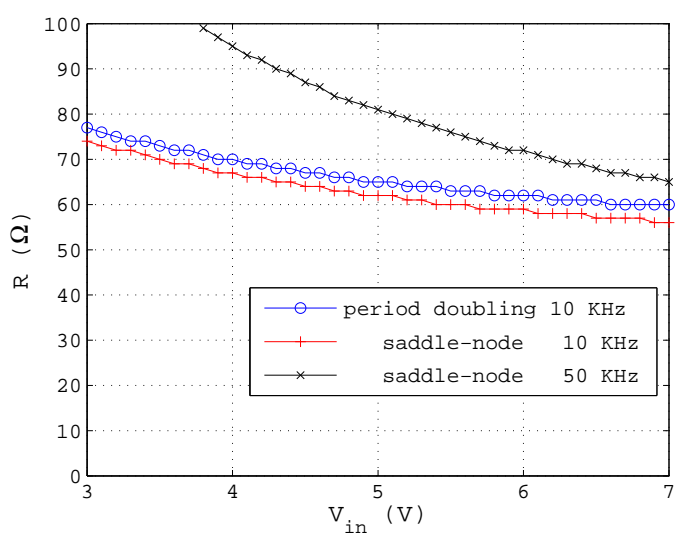

Figure 6: Exact switched model $V_{i n}-R$ bifurcation diagram for gain $\mathbf{k}_{2}=$ $[0.0443,-0.2324]^{T}$. Period doubling vs multiple equilibria curves are shown for $f_{s}=10,50 \mathrm{kHz}$.

or stabilizing control-, which is restricted to a single gain $\gamma>0$ design, and takes the form

$$
u=\mathbf{k}^{T} \mathbf{x}+u_{s s}, \mathbf{k}=\gamma\left[I_{s s}-V_{s s}\right]^{T}, \gamma>0
$$

In this paper, we carry out a novel analysis for the more general case of a feedback controller with two independent gain values $\mathbf{k}=\left[k_{1}, k_{2}\right]^{T} \in \mathbb{R}^{2 \times 1}$. This is the general formulation used in many recent works in power converters for designing robust and optimal control schemes. The first step is to find the expression for the equilibrium points $\left(v_{e q}, i_{e q}\right)$. To do that we use (12) into (7), equate the state derivative to zero and solve for $v_{e q}$. Furthermore, we assume that the controller uses the value $R$ as output load value in the control law specified by (9),(12), while the real output load value is $R_{0}$. This results in a cubic equation $f\left(v_{e q}\right)=0$ with real coefficients that may give one to three real equilibria, where

$$
f\left(v_{e q}\right)=v_{e q}^{3}+r \cdot v_{e q}^{2}+p \cdot v_{e q}+q
$$

and

$r=\frac{V_{i n} k_{1} R}{k_{2}}, p=-\frac{R}{R_{0}} V_{s s}^{2}-R \frac{V_{i n}\left(k_{1} V_{s s}^{2}+V_{i n}\right)}{V_{s s} k_{2}}, q=R \frac{V_{i n}^{2}}{k_{2}}$

We consider equilibria voltages $V_{s s}>V_{\text {in }}$ and feedback gains satisfying $k_{1}>0, k_{2}<0^{4}$ and we have the following definition :

Definition 1 A bilinear system (7) with one, two or three real equilibria is denoted as $E Q 1, E Q 2$ and $E Q 3$, respectively.

Next, we consider two separate cases, depending on the presence or the absence of a controller mismatch.

\footnotetext{
${ }^{4}$ As it is explained in section 5 (and in [18]), this choice of gains results in a stable system with high damping.
} 


\subsection{Without controller's mismatch}

In this case we have $R=R_{0}$ and we define the function $\Gamma\left(V_{s s}, V_{i n}, R, k_{1}, k_{2}\right)=V_{s s}^{3} k_{2}^{2}+V_{s s} V_{i n}^{2} R^{2} k_{1}^{2}+4 R V_{i n}^{2} k_{2}+$

$$
2 V_{\text {in }} R V_{s s}^{2} k_{1} k_{2} \text {. }
$$

Proposition 1 In the absence of controller mismatch, the bilinear system (7) controlled by a state-feedback law (12) exhibits one to three real equilibria and it is

1. EQ 1 if and only if $\Gamma<0$

2. EQ 2 if and only if $\Gamma=0$

3. EQ 3 if and only if $\Gamma>0$

PROOF In the absence of controller mismatch it becomes obvious from (18) that $v_{e q}=V_{s s}$ is always one real equilibrium of (17) so that the cubic $f\left(v_{e q}\right)$ can be further expressed as

$$
f\left(v_{e q}\right)=\left(v_{e q}-V_{s s}\right)\left(v_{e q}^{2}+p_{1} \cdot v_{e q}+q_{1}\right)
$$

where

$$
p_{1}=V_{s s}+R \frac{V_{i n} k_{1}}{k_{2}}, q_{1}=-R \frac{V_{i n}^{2}}{V_{s s} k_{2}}
$$

The discriminant $\Delta_{1}$ of the above quadratic term is given by

$$
\Delta_{1}=\left(V_{s s}+R \frac{V_{i n} k_{1}}{k_{2}}\right)^{2}+4 R \frac{V_{i n}^{2}}{V_{s s} k_{2}}
$$

and may be expressed as a new quadratic in terms of $R$, i.e.

$$
\Delta_{1}=\frac{V_{i n}^{2} k_{1}^{2}}{k_{2}^{2}} \cdot\left(R^{2}+\frac{2 k_{2}\left(k_{1} V_{s s}^{2}+2 V_{i n}\right)}{V_{i n} V_{s s} k_{1}^{2}} R+\frac{k_{2}^{2} V_{s s}^{2}}{k_{1}^{2} V_{i n}^{2}}\right)
$$

The discriminant $\Delta_{2}$ of the latter quadratic is given by

$$
\Delta_{2}=\frac{16 k_{2}^{2}\left(k_{1} V_{s s}^{2}+V_{i n}\right)}{V_{i n} V_{s s}^{2} k_{1}^{4}}
$$

and is always positive since $k_{1}>0, k_{2}<0, V_{\text {in }}>0, V_{s s}>0$.

Therefore, there always exist two real solutions $R_{1}<R_{2}$ of $\Delta_{1}=0$ such that $\Delta_{1} \leq 0$ for $R \in\left[R_{1}, R_{2}\right]$ and $\Delta_{1}>0$ otherwise. This implies that there are two complex solutions of the quadratic term in (19) for $R_{1}<R<R_{2}$, and two real ones otherwise. Hence, for $R_{1}<R<R_{2}$ the cubic equation has a single equilibrium, while otherwise three real equilibria are present (two equal real equilibria are obtained when $\Delta_{1}=0$, i.e. for $R=R_{1}$ or $R=R_{2}$ ). The equation $\Delta_{1}=0$ is the border between the two qualitatively different situations, i.e. it is a multiple equilibria bifurcation curve ${ }^{5}$. Trivial manipulations reveal that $\Delta_{1}=0$ if and only if $\Gamma=0$ and the proof is concluded.

Bifurcation phenomena are pictorially presented with the help of the so-called bifurcation diagrams. Herein we utilize the $V_{i n}-R$ (parameter space) and $k_{1}-k_{2}$ (controller gain space) diagrams. We proceed with some further results in the following lemmas. The proofs can be found in the Appendix.

\footnotetext{
${ }^{5} \mathrm{~A}$ similar analysis can be carried out to express $\Delta_{1}$ as another quadratic in terms of $V_{i n}$, and it can be similarly shown that there always exist two real solutions $E_{1}<E_{2}$ of $\Delta_{1}=0$, so that we have a single equilibrium for any $E_{1}<V_{i n}<E_{2}$, two for $V_{i n}=E_{1}$ or $V_{i n}=E_{2}$ and three equilibria otherwise.
}

Lemma 1 If the converter's operating conditions satisfy

$$
\frac{k_{1} V_{s s}^{2}}{4 V_{s s}^{2} k_{1}^{4}-1}<V_{i n}<\left(\frac{V_{s s}^{2}}{2 k_{1}^{3 / 2}}\right)^{3 / 2}
$$

then the bifurcation curve $\Gamma=0$ on the first quadrant of the $V_{\text {in }}-R$ plane is made of two separate non-intersecting curves dividing the quadrant into three disjoint areas.

Lemma 2 In any converter's operating conditions, the bifurcation curve $\Gamma=0$ on the fourth quadrant of the $k_{1}-k_{2}$ plane is made of two separate non-intersecting curves dividing the quadrant into three disjoint areas.

The properties highlighted in Lemmas 1,2 are shown in Fig. 7 for the converter introduced in subsection 2.3. A representative $k_{1}-k_{2}$ bifurcation diagram is depicted in Fig. 7(a) for $V_{\text {in }}=5 \mathrm{~V}, R=40 \Omega, V_{s s}=10 \mathrm{~V}$, which can be shown to satisfy (24). Likewise, a representative $V_{i n}-R$ bifurcation diagram with similar properties is seen in Fig. 7(c) for $V_{s s}=10$ $\mathrm{V}$ and gain $\mathbf{k}_{2}=[0.0443,-0.2324]^{T}$. The three different areas formed by the two bifurcation curves are clearly seen. The area enclosed by the two curves is marked as "EQ 1 " to denote the existence of a single equilibrium point, whereas the other two areas are marked as "EQ 3".

Imposing conditions to ensure the absence of any bifurcation phenomena can be very restrictive - e.g. a Lyapunov-based controller (16) with a single tuning parameter, i.e. a single degree of freedom can be used -. Less conservative conditions which ensure the absence of any multiple equilibria inside a specific region of interest may be found. E.g. simple state constraints for the output voltage $0 \leq v_{C} \leq V_{C}^{+}$may be included.

Lemma 3 A sufficient condition for the absence of positive multiple real equilibria of (17), in any converter's operating condition, is the satisfaction of the following inequality

$$
\left|\frac{k_{1}}{k_{2}}\right|<\frac{V_{s s}}{R^{+} \cdot V_{i n}^{+}}
$$

Lemma 4 A necessary and sufficient condition for the absence of positive multiple real equilibria of (17) in the interval $v_{C} \leq$ $V_{C}^{+}$, is the satisfaction of the following inequality for the whole range of converter's operating conditions

$$
\left(R V_{i n} V_{s s} V_{C}^{+}\right) k_{1}+\left(V_{s s}+V_{C}^{+}\right) V_{s s} V_{C}^{+} k_{2}-R V_{i n}^{2}<0
$$

The results of Lemmas 3,4 can be combined to guide the control design procedure so that the absence of any positive multiple real equilibria of (17) in the interval of interest $0 \leq$ $v_{C} \leq V_{C}^{+}$is guaranteed. To this end, either (25) or (26) should hold for the whole range of the converter's operating conditions.

\subsection{With controller's mismatch}

Let us assume now that there is a mismatch between the controller and the real system, i.e. the controller uses an output load value $R$ different from the system's load $R_{0}$, i.e. $R \neq R_{0}$. We have the following result. 


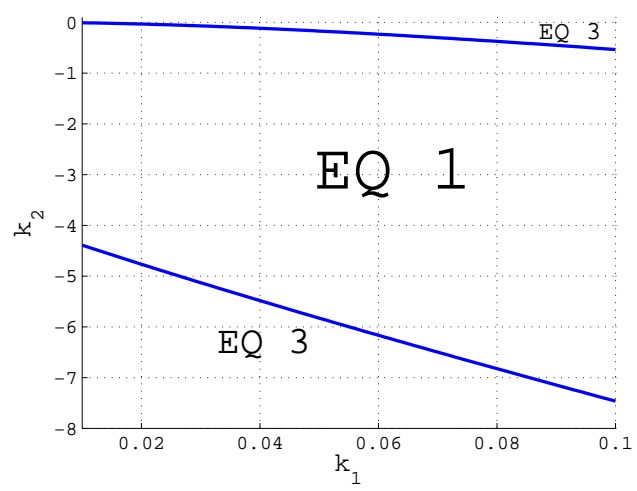

(a)

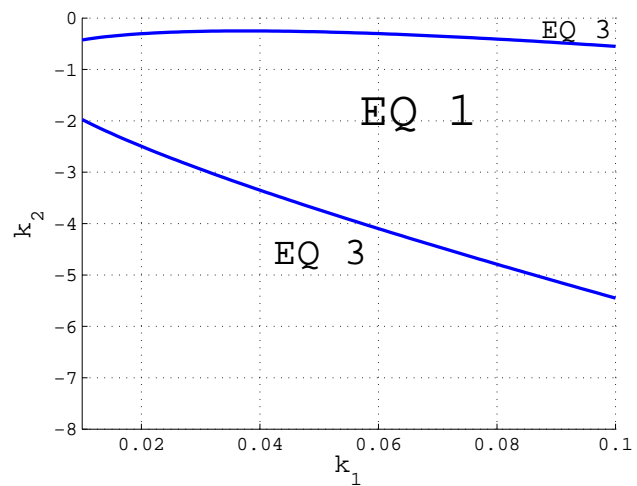

(b)

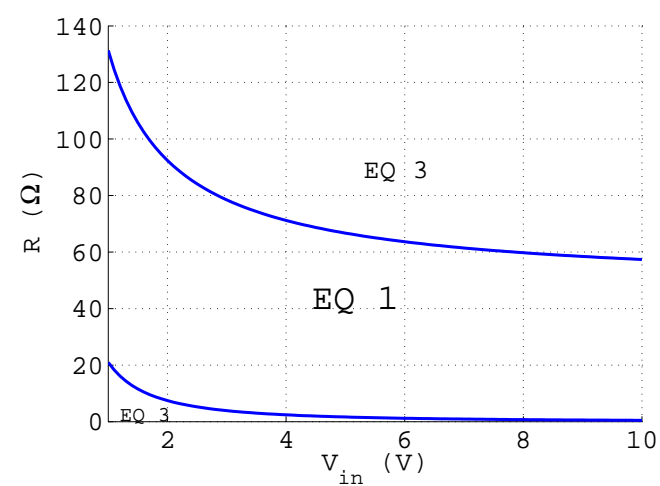

(c)

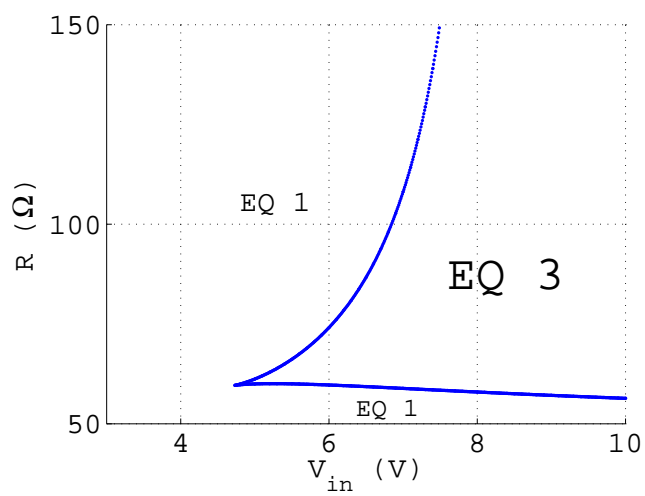

(d)

Figure 7: Representative bifurcation diagrams for the converter of subsection 2.3 with $V_{s s}=10 \mathrm{~V}$. First, $k_{1}-k_{2}$ diagrams with $V_{i n}=5 \mathrm{~V}, R=40 \Omega$ (a) in the absence, and (b) in the presence of controller mismatch are shown. Second, $V_{i n}-R$ diagrams for gain $\mathbf{k}_{2}=[0.0443,-0.2324]^{T}$ (c) in the absence, and (d) in the presence of controller mismatch are compared.
Proposition 2 In the presence of controller mismatch, the bilinear system (7) controlled by a state-feedback law (12) exhibits one to three real equilibria and it is

1. EQ1 if and only if $\Delta<0$

2. EQ2 if and only if $\Delta=0$

3. EQ3 if and only if $\Delta>0$

where $\Delta$ is defined as

$$
\Delta=4\left(\frac{V_{i n}}{V_{s s} k_{1}}+V_{s s}+\frac{V_{s s}^{2} k_{2}}{V_{i n} R_{0} k_{1}}\right)^{2}-\frac{12 V_{i n}}{k_{1}}
$$

PROOF In the presence of controller mismatch we cannot carry out the same analysis as before, since an equilibrium equal to the desired value $V_{\text {ref }}=V_{s s}$ does not exist any more. We follow a different path, i.e. assuming $R_{0}$ is known, we solve from the cubic (17) for $R$ and express it as a function of the unknown $v_{e q}$

$$
R=R\left(v_{e q}\right)=\frac{-\mu \cdot v_{e q}^{3}}{\nu \cdot v_{e q}-\xi \cdot v_{e q}^{2}-\pi}
$$

where

$\mu=-R_{0} V_{s s} k_{2}>0, \nu=k_{2} V_{s s}^{3}+R_{0} k_{1} V_{i n} V_{s s}^{2}+R_{0} V_{i n}^{2}$

and

$$
\xi=R_{0} k_{1} V_{i n} V_{s s}>0, \pi=R_{0} V_{i n}^{2} V_{s s}>0
$$

Its derivative w.r.t. $v_{e q}$ is given by

$$
\frac{d R}{d v_{e q}}=\frac{\mu \cdot \xi \cdot v_{e q}^{2}}{\left(\nu \cdot v_{e q}-\xi \cdot v_{e q}^{2}-\pi\right)^{2}}\left(v_{e q}^{2}-2 \frac{\nu}{\xi} \cdot v_{e q}+\frac{3 \mu}{\xi}\right)
$$

By setting the derivative equal to zero we end up with a quadratic in $v_{e q}$ with discriminant equal to $\Delta$, which is a parabola in the $k_{1}-k_{2}$ space $^{6}$. A corresponding bifurcation curve $\Delta=0$ is defined, whose sign determines the number of the real equilibria.

In the areas where $\Delta<0$ there are no extremum points for $R\left(V_{s s}\right)$, hence we have a single equilibrium point. If $\Delta>0$ then there are two real roots $v_{1}, v_{2}$ s.t. the derivative is negative in the interval $\left[v_{1}, v_{2}\right]$ and positive otherwise. This implies that the function $R\left(V_{s s}\right)$ is monotonically increasing for $V_{s s}<v_{1}$ or $V_{s s}>v_{2}$ and monotonically decreasing for $v_{1}<V_{s s}<v_{2}$. It is also $R(0)=0$.

The two solutions $v_{1}, v_{2}$ are given by

$$
v_{1,2}=\frac{\nu}{\xi} \pm \sqrt{\left(\frac{\nu}{\xi}\right)^{2}-\frac{3 \mu}{\xi}}
$$

and their sign depends on the value of $\nu$.

If $\nu>0$, both roots are positive, and since $R(0)=0$ and the derivative is positive for values $V_{s s}<v_{1}$ we have

${ }^{6}$ It is $B^{2}-4 A C=0$ if $\Delta$ is written in the form $\Delta=A k_{1}^{2}+B k_{1} k_{2}+$ $C k_{2}^{2}+D k_{1}+E k_{2}+F=0$. 
$R_{1}=R\left(v_{1}\right)>0$. Otherwise, if $\nu<0$, both roots are negative, the phenomena appear in an interval corresponding to negative values for $V_{s s}$ which is unrealistic and can be therefore ignored. In any case, when three real equilibria exist, they appear in the interval $R \in\left[R_{1}, R_{2}\right]$, where $R_{1}=R\left(v_{1}\right), R_{2}=R\left(v_{2}\right)$.

For the same data used before, we present new bifurcation diagrams in Fig. 7(b),(d), in the presence of controller mismatch. By comparing the two $k_{1}-k_{2}$ bifurcation diagrams in Fig. 7, we observe that they are both qualitatively similar. However, only a subset of the initial area in Fig. 7(a) remains "EQ 1 ", i.e. the area in the $k_{1}-k_{2}$ space corresponding to a single equilibrium is clearly reduced in size. This result indicates that, in the presence of controller mismatch, multiple equilibria phenomena occur for a wider variety of feedback gains.

Furthermore, in the presence of controller mismatch, a qualitatively different $V_{i n}-R$ bifurcation diagram is obtained. For the same controller gain used in Fig. 7(c), the new diagram is shown in Fig. 7(d), where the single equilibrium region is now the area outside the V-shaped region. This is confirmed by the previous analysis, in which it was proved that multiple equilibria are born for output load $R$ values in the interior of the interval $\left[R_{1}, R_{2}\right]$-for which two real solutions $v_{1}, v_{2}$ exist. This fact is justified by studying the representative picture of the function $R=R\left(V_{s s}\right)$ shown in Fig. 8, for a fixed value of $R_{0}=40 \Omega$ as before and different $V_{i n}$ values, in the presence of controller mismatch.

More specifically, for the same data as before, we consider three different values of $V_{i n}$ in order to explain the shape of the area found in Fig. 7(d). For $V_{i n}=3.5 \mathrm{~V}$, we obtain a monotonically increasing curve, hence the existence of a single equilibrium is assured. Moreover, this curve allows us to observe how this equilibrium is moved away from its desired position as $R \neq R_{0}$ is increased. We see that for a $50 \%$ increase from $R=40 \Omega$ to $R=60 \Omega$ the single equilibrium's steady-state output voltage is almost doubled from $10 \mathrm{~V}$ to $20 \mathrm{~V}$, while for values $R \cong 70 \Omega$ it is already tripled.

For $V_{i n}=5 \mathrm{~V}$, two real solutions $v_{1}, v_{2}$ are marginally obtained, with the corresponding load values being very close to each other, i.e. $R_{1}=59.97, R_{2}=61.21 \Omega$. For a further increase to $V_{\text {in }}=6.5 \mathrm{~V}$, a larger interval is obtained for which three distinct equilibria exist. The values $R_{1}=59.30 \Omega, R_{2}=$ $86.67 \Omega$ are found in this case, and it is clear from the graph's shape that for any value $R \in\left[R_{1}, R_{2}\right]$ three corresponding values of $V_{s s}$ can be obtained. An example is shown in Fig. 8, where for $R=66.63 \Omega$ three distinct values $v_{1}=10.5 \mathrm{~V}$, $v_{2}=21 \mathrm{~V}, v_{3}=50 \mathrm{~V}$ are specified. The results in Fig. 8 are in perfect agreement and justify the $\mathrm{V}$-shaped area found in Fig. 7(d).

It is interesting to check whether the results found using the exact switched model for the saddle-node bifurcation agree with those obtained by the averaged model. For two different gains, the $V_{i n}-R$ bifurcation diagram produced by the analysis of the previous section, for the averaged model, is presented in Fig. 9. Comparison of the curves in Figs. 6 and 9 for the gain $\mathbf{k}_{2}=[0.0443,-0.2324]^{T}$ reveals a close resemblance and justifies the predictions made by our bifurcation analysis

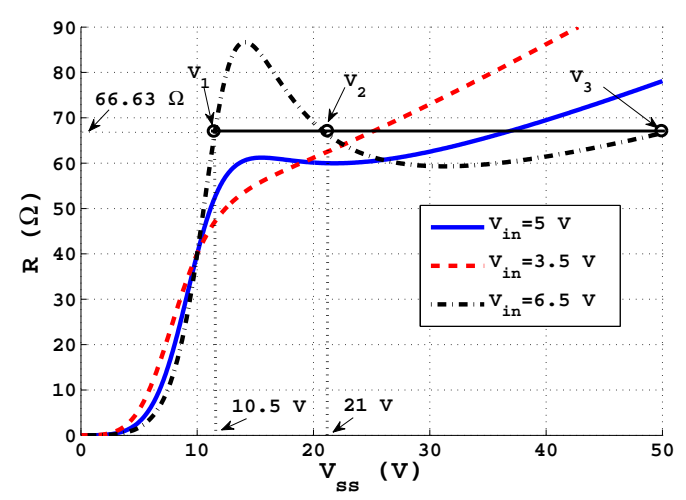

Figure 8: The function $R=R\left(V_{s s}\right)$ for $R_{0}=40 \Omega$ and varying $V_{i n}$, in the presence of controller mismatch, for gain $\mathbf{k}_{2}=[0.0443,-0.2324]^{T}$.

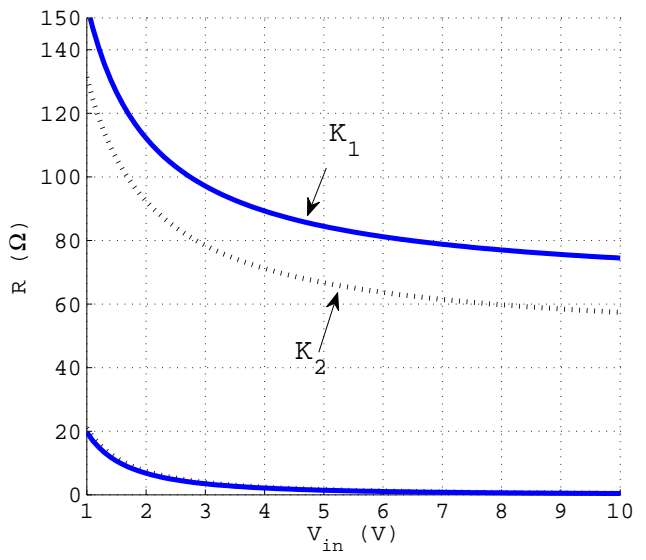

Figure 9: $V_{i n}-R$ bifurcation diagrams using the averaged model for gains $\mathbf{k}_{1}=[0.043,-0.2825]^{T}$ and $\mathbf{k}_{2}=[0.0443,-0.2324]^{T}$ for $V_{\text {ref }}=10 \mathrm{~V}$.

on the basis of the averaged model. The effect of the feedback gain is also clearly shown in Fig. 9. Although the same phenomena cannot be avoided, a slightly different feedback gain $\mathbf{k}_{1}=[0.043,-0.2825]^{T}$ can shift them outside of the interval of interest. The averaged-model results are again in close agreement with the result obtained with the switched model.

Finally, it is worth mentioning that all the analysis results found in this and the previous section justify the simulation results presented in subsection 2.3. We have observed that predictions using the averaged model are sound and accurate, hence the bifurcation avoidance criteria obtained in section 3.1 could be used to guide the control design process.

\section{Control design through bifurcation analysis and constrained stabilization}

The problem of designing controllers for the converter's system (7) can be faced as a constrained stabilization problem. Along these lines, as it is common to design controllers to achieve regulation around the zero state, it is natural to apply a linear transformation, so that the non-zero equilibrium state is mapped to the zero state in the new transformed state-space. 
We consider as new state-space variables the error variables $x_{e}$ and input $u_{e}$ such that

$$
\mathbf{x}_{e}=\mathbf{x}-\mathbf{x}_{s s} \text { and } u_{e}=u-u_{s s}
$$

to arrive from $(7),(8)$ at a new auxiliary bilinear system in the form

$$
\dot{\mathbf{x}}_{e}=f\left(\mathbf{x}_{e}, u_{e}\right)=\mathbf{A} \mathbf{x}_{e}+\mathbf{B}_{1} \mathbf{x}_{e} u_{e}+\mathbf{B}_{2} u_{e}
$$

where

$$
\mathbf{A}=\mathbf{A}_{1}+\mathbf{A}_{2} u_{s s}, \mathbf{B}_{1}=\mathbf{A}_{2}, \mathbf{B}_{2}=\mathbf{A}_{2} \mathbf{x}_{s s}
$$

For the transformed input, hard limits $u_{e} \in \mathcal{U}$ are imposed, where $\mathcal{U}$ is the unsaturated region (URE) defined as

$$
\mathcal{U}=\left\{u_{e} \in \mathbb{R}:-u_{s s} \leq u_{e} \leq 1-u_{s s}\right\}
$$

Moreover, specific a priori known constraints on the converter's voltages and currents are introduced for safety reasons and define the state constraint set $\mathcal{X}$

$$
\mathcal{X}=\left\{\mathbf{x} \in \mathbb{R}^{2}: V_{C}^{-} \leq v_{C} \leq V_{C}^{+}, I_{L}^{-} \leq i_{L} \leq I_{L}^{+}\right\}
$$

We consider control laws in an affine state-feedback form, which for a non-zero equilibrium $\mathbf{x}_{s s}$ and corresponding input $u_{s s}$ are stated as

$$
u=\mathbf{k}^{T}\left(\mathbf{x}-\mathbf{x}_{s s}\right)+u_{s s} \text { or } u_{e}=g\left(\mathbf{x}_{e}\right)=\mathbf{k}^{T} \mathbf{x}_{e}
$$

This is a standard form of an affine state-feedback control law, with no direct incorporation of integral action. The controller consists of a feedback and a feed-forward term. The feedback gain is fixed and time-invariant. The feed-forward term is a parameter-dependent offset, which depends on the operating point changes and is calculated on-line on the basis of an estimator. Such estimators (observers) of the unmeasurable disturbances (input voltage and/or output load) have been also used in relevant works, and can take the form of a moving-average filter [19], a sliding mode observer [20] or a Kalman filter [4].

In our setting it is not sufficient to justify that the origin of the state space is locally asymptotically stable, but also to make sure that the operating range is included into the region of attraction of the equilibrium. We have the following definition:

Definition 2 A subset $\mathcal{C}$ of $\mathcal{X}$ is an unsaturated domain of attraction (UDOA) for a given equilibrium point $\mathbf{x}_{e} \in \operatorname{ri}\{\mathcal{C}\}$ of system (34), if for all $\mathbf{x} \in \mathcal{C}$ it holds that $g\left(\mathbf{x}_{e}\right) \in \mathcal{U}$ and $f\left(\mathbf{x}_{e}, u_{e}\right) \in \mathcal{C}$ and all trajectories are asymptotically attracted to the desired equilibrium, i.e. $\lim _{t \rightarrow \infty} f\left(\mathbf{x}_{e}, u_{e}\right)=0$.

The efficient construction of maximal UDOAs is of fundamental importance in any control design process based on constrained stabilization principles. Constrained stabilization techniques rely on the construction of invariant and contractive sets in the state-space and corresponding set-induced Lyapunov functions, while all input and state constraints, bounded parameter uncertainties, nonlinear dynamics and optimal performance are also addressed. To this end, flexible piecewise linear
(PL) Lyapunov functions proposed recently in [17] are utilized, which rely on ray-gridding as a systematic technique for dealing with low dimensional PL systems via the construction of UDOAs using PL Lyapunov functions. The technique has been applied successfully to several different linear switched system analysis and design problems in the past $[21,22,23]$.

\subsection{A new control design procedure}

The efficient construction of maximal unsaturated domains of attraction (UDOAs) may be performed through constrained stabilization principles. The usual practice is to combine the control law synthesis with the contractive set construction, which is performed using appropriate iterative algorithms. All raygridding algorithms operate with a special emphasis on maximal set size. In other algorithms, fixed predetermined sets are considered and control laws offering optimal performance are synthesized. Recently, similar iterative algorithms have been proposed [24],[6] for the concurrent synthesis of state-feedback laws and contractive sets, which are modified in an iterative manner to achieve the maximum possible enlargement of the set.

Unfortunately, for the more demanding problem considered in this work, i.e. robust tracking in the large, the traditional synthesis of state-feedback control laws on the basis of a single contractive set for a specific operating condition is insufficient, since no direct parametrization for varying uncertain parameters is possible. This is obvious from (34),(35), where it becomes clear that the bilinear system matrices involved are equilibrium point dependent in a nonlinear manner. Subsequently, systematic construction of families of contractive sets covering the whole range of operating condition is necessary. In this case, the synthesis of control laws cannot be done w.r.t. a single contractive set alone : an efficient technique for synthesizing in an optimal (or suboptimal) manner control laws that ensure a whole family of contractive sets simultaneously are needed. Such a technique has to be capable of producing sufficient large (near-maximal) domains -when optimality w.r.t. set size is important-, as well as appropriate trade-offs between contractive sets size and optimal performance -when performance-related aspects, e.g. certain contractivity rate demands are important-.

Furthermore, for switched-mode converter applications, undesirable bifurcation phenomena with unstable attractors are possible, and special care for their avoidance must be taken during the design procedure. Unfortunately, the previously mentioned techniques do not provide the means to incorporate bifurcation avoidance conditions a priori in the design process. This may require significant a posteriori testing with bifurcation analysis, which makes the design an iterative process that may involve multiple trials, without clear and systematic guidelines.

A new control law synthesis technique is adopted assuming that the whole range of operating conditions is a-priori known. First, the feasible region in the control gains space is specified such that a number of important requirements and specifications are satisfied, including special bifurcation avoidance conditions. Second, a control law is selected in an optimal manner, 
i.e. by maximizing performance related metrics. Finally, the control law selected is validated by constructing a sufficiently dense family of contractive near-maximal domains covering the whole operating range. If the performance of the proposed controller is not satisfactory, this framework allows flexible and transparent re-designs with new specifications to be performed, giving rise to different trade-offs between conflicting goals.

\subsubsection{Hopf bifurcation boundary}

The occurrence of a Hopf bifurcation can be easily addressed using the well-known Routh-Hurwitz criterion for the linear part of the boost converter bilinear dynamics, i.e. the linearized approximation around the equilibrium point. From (34) the linearized closed-loop model becomes

$$
A_{l i n}=\left[\begin{array}{cc}
-\frac{1}{R C}-\frac{1}{C} \cdot I_{s s} \cdot k_{1} & \frac{1}{C} \cdot\left(1-d_{s s}-I_{s s} k_{2}\right) \\
-\frac{1}{L} \cdot\left(1-d_{s s}-V_{s s} k_{1}\right) & \frac{1}{L} V_{s s} \cdot k_{2}
\end{array}\right]
$$

For a $2 \times 2$ matrix $M=\left[\begin{array}{ll}m_{11} & m_{12} \\ m_{21} & m_{22}\end{array}\right]$ the Routh-Hurwitz criterion for stability requires $m_{11}+m_{22}<0, m_{11} m_{22}-$ $m_{12} m_{21}>0$. By substituting $d_{s s}=1-\frac{V_{i n}}{V_{s s}}$ and $I_{s s}=\frac{V_{s s}^{2}}{R V_{i n}}$ to (39) we have

$$
A_{l i n}=\left[\begin{array}{cc}
-\frac{1}{R C} \cdot\left(1+\frac{V_{s s}^{2}}{V_{i n}} \cdot k_{1}\right) & \frac{1}{C} \cdot\left(\frac{V_{i n}}{V_{s s}}-\frac{V_{s s}^{2}}{R V_{i n}} \cdot k_{2}\right) \\
\frac{1}{L} \cdot\left(V_{s s} \cdot k_{1}-\frac{V_{i n}}{V_{s s}}\right) & \frac{V_{s s}}{L} \cdot k_{2}
\end{array}\right]
$$

Application of the stability criterion gives

$$
\begin{gathered}
-\frac{V_{s s}^{2}}{R C V_{i n}} \cdot k_{1}+\frac{V_{s s}}{L} \cdot k_{2}-\frac{1}{R C}<0 \\
\frac{V_{i n}}{L C} \cdot k_{1}+2 \frac{V_{s s}}{R L C} \cdot k_{2}-\frac{V_{i n}^{2}}{L C V_{s s}^{2}}<0
\end{gathered}
$$

Since we restrict our attention to gains $k_{1}>0, k_{2}<0$, the first equation (41) is trivially satisfied for any values of the uncertain parameters (11), whereas (42) is satisfied for all values if and only if the maximum w.r.t. to $R$ is negative, i.e.

$$
\frac{V_{i n}}{L C} \cdot k_{1}+2 \frac{V_{s s}}{R^{+} L C} \cdot k_{2}-\frac{V_{i n}^{2}}{L C V_{s s}^{2}}<0
$$

\subsubsection{Performance specifications}

Simple time-domain performance specifications in terms of the linearized model can be also easily set on the basis of typical settling time and overshoot bounds.

For a $2 \times 2$ matrix $M=\left[\begin{array}{ll}m_{11} & m_{12} \\ m_{21} & m_{22}\end{array}\right]$ the eigenvalues quadratic equation $\left|s \cdot I_{2}-M\right|=s^{2}+2 \cdot \zeta \cdot \omega_{n} s+\omega_{n}^{2}=0$ implies that $m_{11}+m_{22}=-2 \cdot \zeta \cdot \omega_{n}, m_{11} m_{22}-m_{12} m_{21}=\omega_{n}^{2}$.

A settling time requirement $T_{s}<T_{d}$, where $T_{d}$ a minimum desired time bound, can be expressed as $-m_{11}-m_{22}>\frac{8}{T_{d}}$.

Similarly, a minimum overshoot bound may be set by imposing $\zeta>\zeta_{d}$, where $\zeta_{d}$ a minimum acceptable overshoot, and may be expressed as $m_{11} m_{22}-m_{12} m_{21}<\omega_{d}^{2}$, where $\omega_{d}=\frac{4}{T_{d} \zeta_{d}}$.
Substituting for the entries of matrix $M$ from (40) leads to

$$
\begin{gathered}
\frac{V_{s s}^{2}}{R C V_{i n}} \cdot k_{1}-\frac{V_{s s}}{L} \cdot k_{2}+\frac{1}{R C}-\frac{8}{T_{d}}>0 \\
0<-\frac{V_{i n}}{L C} \cdot k_{1}-2 \frac{V_{s s}}{R L C} \cdot k_{2}+\frac{V_{i n}^{2}}{L C V_{s s}^{2}}<\omega_{d}^{2}
\end{gathered}
$$

These are satisfied for all values of the uncertain parameters if and only if

$$
\begin{gathered}
\frac{V_{s s}^{2}}{R^{+} C V_{i n}^{+}} \cdot k_{1}-\frac{V_{s s}}{L} \cdot k_{2}+\frac{1}{R^{+} C}-\frac{8}{T_{d}}>0 \\
-\frac{V_{i n}}{L C} \cdot k_{1}-2 \frac{V_{s s}}{R^{-} L C} \cdot k_{2}+\frac{V_{i n}^{2}}{L C V_{s s}^{2}}<\omega_{d}^{2}
\end{gathered}
$$

\subsubsection{Saturation avoidance criteria}

The problem of selecting controller gains that ensure saturation avoidance for the whole operating range may be transformed to a simple geometrical set inclusion problem. More specifically, it is enough to ensure that the unsaturated region includes the feasible equilibria region (FER) as in (10).

To explain this further, consider the equilibrium point given by (9) for varying parameters $V_{i n}, R$. When the converter operates around the nominal operating region, the equilibrium point stays within a small neighborhood of the equilibrium $\mathbf{x}_{0}$ (obtained from (9) for nominal parameter values). However, when the uncertain parameters $V_{i n}, R$ vary within a wide range, the FER grows significantly. This can be clearly seen in Fig. 10, for data taken from the example in section 5 , where $\mathbf{x}_{0}=\left[\begin{array}{ll}10 & 0.5\end{array}\right]^{T}$. When any one of the uncertain parameters, e.g. the load is switched to a new value, a new equilibrium point is generated and the old equilibrium becomes the initial condition for the transient that will follow. To ensure that the output voltage will always be driven to the new equilibrium point without any saturation phenomena we must guarantee that any (possible) initial condition will be included in the UDOA of the new equilibrium.

The unsaturated region is the area enclosed by the two saturation lines $u=0$ and $u=1$. For the general form of the controller expression $u=\mathbf{k}^{T}\left(\mathbf{x}-\mathbf{x}_{s s}\right)+d_{s s}$ these lines are expressed as $\mathbf{k}^{T}\left(\mathbf{x}-\mathbf{x}_{s s}\right)=-d_{s s}, \mathbf{k}^{T}\left(\mathbf{x}-\mathbf{x}_{s s}\right)=1-d_{s s}$ and their distances from the equilibrium point are

$$
d_{1}\left(\mathbf{k}, d_{s s}\right)=\frac{1}{\sqrt{\mathbf{k}^{T} \mathbf{k}}} d_{s s}, \quad d_{2}\left(\mathbf{k}, d_{s s}\right)=\frac{1}{\sqrt{\mathbf{k}^{T} \mathbf{k}}}\left(1-d_{s s}\right)
$$

The problem of specifying those gains that ensure an unsaturated region covering the feasible equilibria is a set inclusion condition, i.e. we have to make sure that the FER is a subset of the unsaturated region.

In our case, for a fixed reference $V_{s s}$ and variable $V_{i n}, R$ as in (11), the FER reduces to a line segment

$$
\mathcal{L}=\left\{\mathbf{x} \mid x_{1}=V_{s s}, I^{-} \leq x_{2} \leq I^{+}\right\}
$$

with extreme points $\mathbf{p}_{1}=\left[V_{s s}, I^{+}\right]^{T}, \mathbf{p}_{2}=\left[V_{s s}, I^{-}\right]^{T}$, where $I^{-}=V_{s s}^{2} /\left(R^{+} V_{i n}^{+}\right), I^{+}=V_{s s}^{2} /\left(R^{-} V_{i n}^{-}\right)($see e.g. Fig. 10 in 
the next section). Then it can be easily shown that, for saturation avoidance, it is necessary and sufficient to ensure that both extreme points, used as equilibrium points, have large enough distances to include the FER.

For a pair of points $\left(\mathbf{p}_{1}, \mathbf{p}_{2}\right)$, if $\mathbf{k}^{T} \cdot \mathbf{p}_{1}=c_{1}$ and $\mathbf{k}^{T} \cdot \mathbf{p}_{2}=$ $c_{2}$, the distance between the point $\mathbf{p}_{1}$ and the line $\mathbf{k}^{T} \cdot \mathbf{p}_{2}=c_{2}$ may be expressed as

$$
d_{12}=\frac{1}{\sqrt{\mathbf{k}^{T} \cdot \mathbf{k}}} \cdot\left|c_{1}-c_{2}\right|
$$

Hence, a necessary and sufficient condition for saturation avoidance is

$$
d_{12} \leq \min \left[d_{1}\left(\mathbf{k}, d_{s s}^{-}\right), d_{2}\left(\mathbf{k}, d_{s s}^{+}\right)\right]
$$

where $d_{s s}^{-}, d_{s s}^{+}$are the values that correspond to $I^{-}, I^{+}$, and the extreme points $\mathbf{p}_{2}, \mathbf{p}_{1}$, respectively, i.e.

$$
d_{s s}^{-}=1-V_{i n}^{+} / V_{s s}, d_{s s}^{+}=1-V_{i n}^{-} / V_{s s}
$$

For our FER the condition (51) reduces to the simple form

$$
\left|k_{2}\right| \leq \frac{\min \left(d_{s s}^{-}, 1-d_{s s}^{+}\right)}{I^{+}-I^{-}}
$$

In the case of a Lyapunov-based controller as in (16) we have

$$
\gamma \leq \frac{\min \left(d_{s s}^{-}, 1-d_{s s}^{+}\right)}{V_{s s}\left(I^{+}-I^{-}\right)}
$$

\subsubsection{Bifurcation analysis criteria}

The criteria derived in section 3.1 for the avoidance of multiple equilibria can provide valuable guidance to the design process. Imposing conditions to ensure the absence of any bifurcation phenomena can be very restrictive, e.g. a Lyapunov-based controller (16) with a single tuning parameter, i.e. a single degree of freedom can be used. Less conservative conditions have been derived which are easily translated to linear (25) or nonlinear curves (26). As shown next, these curves are very useful for locating feedback gains that keep unstable attractors outside of the region of interest.

\section{An illustrative example}

In this section the motivating example introduced in subsection 2.3 is revisited and the controller design procedure introduced in the previous section is applied. We consider the same boost converter with nominal parameter values $L=1.5 \mathrm{mH}$, $C=10 \mu \mathrm{F}, R=40 \Omega, V_{\text {in }}=5 \mathrm{~V}, V_{\text {ref }}=10 \mathrm{~V}$, for large uncertain variable intervals, i.e. , $V_{\text {in }} \in[3.5,6.5] \mathrm{V}, R \in[20,80] \Omega$, which result in $d_{s s} \in[0.35,0.65]$.

A region of interest $\mathcal{D}$ is selected

$$
\mathcal{D}=\left\{x \in \mathbb{R}^{2}: 0 \leq x_{1} \leq 30,0 \leq x_{2} \leq 1.5\right\}
$$

so that hard safety constraints for the inductor current $0<i_{L} \leq$ $1.5 \mathrm{~A}$ and the capacitor voltage $0 \leq v_{C} \leq 30 \mathrm{~V}$ are respected. Furthermore, we impose the typical saturation avoidance condition for the duty cycle $d \in[0,1]$. Note that these constraints

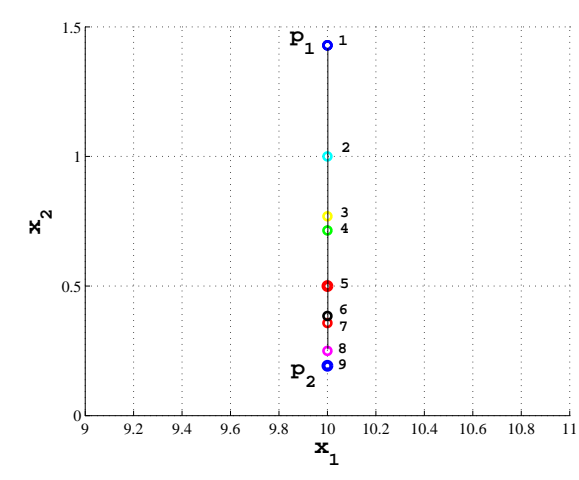

Figure 10: The feasible equilibria region (FER) and the two extreme equilibria points $\mathbf{p}_{1}, \mathbf{p}_{2}$.

\begin{tabular}{cccc}
\multicolumn{4}{c}{ Table 1: Chromatic-numbering code } \\
\hline \hline Index & $V_{\text {in }}$ & $\mathrm{R}$ & Color \\
\hline \hline 1 & 3.5 & 20 & blue \\
\hline 2 & 3.5 & 40 & green \\
\hline 3 & 3.5 & 80 & red \\
\hline 4 & 5 & 20 & cyan \\
\hline 5 & 5 & 40 & thick red \\
\hline 6 & 5 & 80 & magenta \\
\hline 7 & 6.5 & 20 & yellow \\
\hline 8 & 6.5 & 40 & black \\
\hline 9 & 6.5 & 80 & thick blue \\
\hline \hline
\end{tabular}

guarantee that the converter operates in continuous-conduction mode. $^{7}$

With the parameter ranges selected, the open-loop equilibria (9) define the feasible equilibria region (FER) shown in Fig. 10 , where the chromatic and numbering code used is given in Table 1. The FER is a line segment with extreme points $\mathbf{p}_{1}=[10,1.42]$ (for $\left.V_{i n}=3.5 \mathrm{~V}, R=20 \Omega, d_{s s}=0.65\right)$ and $\mathbf{p}_{2}=[10,0.19]$ (for $V_{i n}=6.5 \mathrm{~V}, R=80 \Omega, d_{s s}=0.35$ ).

Selecting large control gains is beneficial in terms of performance, but may result in limited safety, i.e. a small UDOA. On the other hand, selecting lower gains guarantees a larger UDOA. Our approach can be used to search for a single control gain such that a large enough UDOA is obtained for the whole range of operating condition.

\subsection{Control design using the feasible region on the $k_{1}-k_{2}$ plane}

A representative diagram is shown in Fig. 11(a), where five different separating curves have been plotted, according to the main control design requirements and specs explained in section 4 , as follows

1. The curve numbered 1 is the Hopf Bifurcation boundary produced using (43) for all possible values of $V_{i n}$. The

\footnotetext{
${ }^{7}$ It has to be noted here, that due to the presence of a diode in the converter's circuit, the actual inductor current cannot become negative and therefore the averaged current will only become zero if the inductor current is zero during the whole clock cycle. Hence the lower bound value $i_{L}^{-}$should be set to a nonzero value that depends on the current ripple. Having said that, in this work we take it as zero to simplify the resulting expressions.
} 
left subspace of the curve guarantees closed-loop stability.

2. The curve numbered 2 is the natural frequency curve produced using (47) for all possible values of $V_{i n}$. The right subspace of the curve satisfies a minimum $\zeta=0.5$ requirement.

3. The line numbered 3 is the settling time line produced using (46) for $T_{d}=2 \mathrm{msec}$. The lower subspace of the curve satisfies $T_{s} \leq 2 \mathrm{msec}$.

4. The curve numbered 4 is a bifurcation suppression curve obtained from (26) for $V_{C}^{+}=30 \mathrm{~V}$ (the border of the region of interest). The left subspace assures the absence of multiple equilibria inside the region of interest.

5. The line numbered 5 is the saturation avoidance curve produced using (53), for the pair $\left(\mathbf{p}_{1}, \mathbf{p}_{2}\right)$ of extreme points shown in Fig. 10, which reduces to $\left|k_{2}\right| \leq 0.2846$. The upper subspace ensures saturation avoidance.

The diagram shown in Fig. 11(a) is the result of experimenting with different values for $T_{d}, \omega_{d}, \zeta_{d}$ so that a feasible solution guaranteing a sufficiently fast and low overshoot response, in the large (for all values of uncertain parameters) is obtained. It can be shown that for values $\zeta_{d}>0.5$ it is impossible to find any $k_{1}, k_{2}$ values satisfying all other important requirements, since there is no intersection between the corresponding subspaces. For $\zeta_{d}=0.5$, there is a feasible region -specified as the common intersection between the left subspaces of curves $1,3,4$, and the upper subspaces of curves 2,5provided that $T_{d} \geq 2 \mathrm{msec}$, approximately. A close-up of the small feasible region that is generated in this case is shown in Fig. 11(b). Inside this region, an optimal gain choice in terms of smallest possible settling time is

$$
\mathbf{k}_{1}=[0.043,-0.2825]^{T}
$$

\subsection{Verification using constrained stabilization and polyhedral Lyapunov functions}

The last important step is analysis of the design outcome using constrained stabilization principles. The gain selected from the feasible gain space for a desirable design trade-off is validated with the systematic construction of a sufficiently dense family of UDOAs using polyhedral Lyapunov functions. This family can guarantee the converter's safe operation under state and control constraints for the whole operating regime.

Our gain choice $\mathrm{k}_{1}$ as in (56) has been found to keep the bifurcation phenomena outside of the region of interest in previous sections. This is also confirmed in all simulation experiments shown in subsection 2.3 with large disturbances. In order to fully validate this design, the ray-gridding approach [17] has been applied to generate a family of convex and nonsymmetrical polyhedral contractive domains for all operating conditions. Using the same values of the uncertain parameters and the chromatic code of Table 1, we present the results in Fig. 12. This gives us a representative picture of the UDOAs obtained under different operating conditions. A total of 9 different domains are plotted, by taking all combinations of three

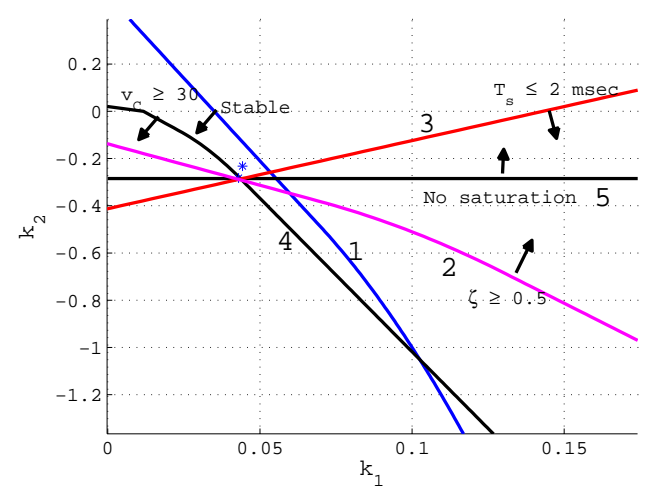

(a)

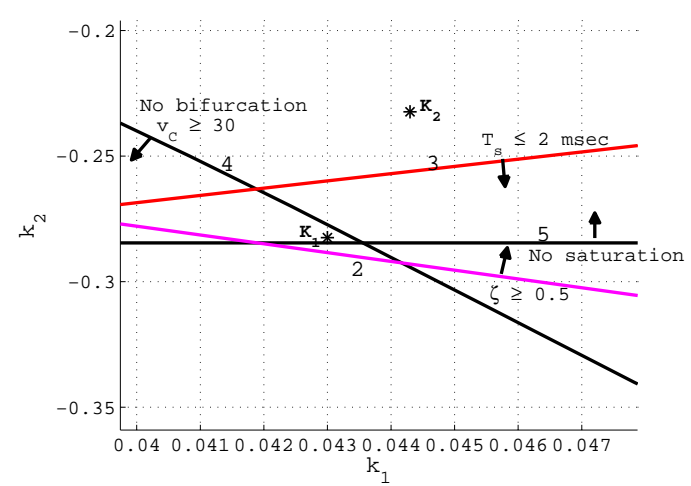

(b)

Figure 11: (a) Complete feasibility conditions in the $k_{1}-k_{2}$ space. The curve numbers are as follows : (1) Hopf bifurcation boundary, (2) $\zeta_{n} \geq 0.5$, (3) $T_{s} \leq 2 \mathrm{msec}$, (4) multiple equilibria position criterion (26) for $V_{C}^{\overline{+}}=30 \mathrm{~V}$, (5) saturation avoidance criterion, (b) A close-up of the small feasible region.

different values (nominal, minimum and maximum) for each of the uncertain parameters $V_{i n}, R$. More specifically,

- Saturation during the startup transient is completely avoided in all cases. All UDOAs in Fig. 12 include the origin, hence the startup transient is saturation-free, without the need to impose any additional rate constraints.

- In all cases, the UDOAs found are such that the FER shown in Fig. 10 is included in their interior. This confirms the usefulness of the set-inclusion criterion described in the previous section.

Remark 1 The design procedure described above on the basis of the feasible gain space is a transparent synthesis technique allowing desirable trade-offs between different performance specifications to be made, provided that saturation avoidance and custom bifurcation avoidance conditions are satisfied, and closed-loop stability is ensured. The saturation avoidance criterion is exact and the bifurcation avoidance conditions are necessary and sufficient, since they are based on the exact bilinear converter dynamics. This is not the case with the stability and performance specifications, which are necessary only (since they are based on the approximated linear model). Hence, the solution found is suboptimal. However, in cases where a 


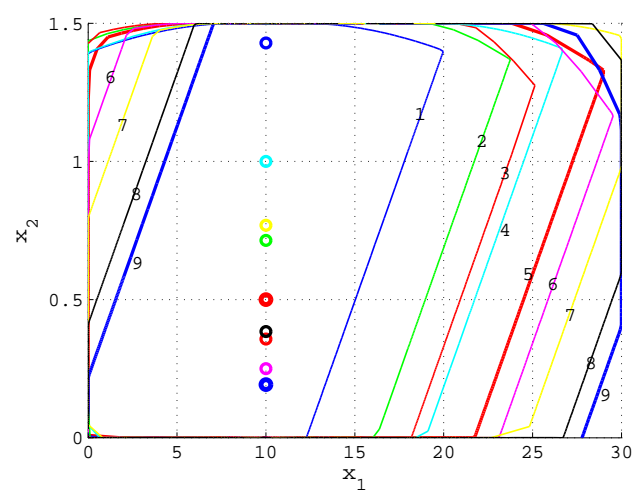

Figure 12: Family of contractive domains for various operating conditions $\left(V_{i n}=3.5,5,6.5 \mathrm{~V}, R=20,40,80 \Omega\right)$ for gain $\mathbf{k}_{1}=[0.043,-0.2825]^{T}$ and $V_{s s}=10 \mathrm{~V}$.

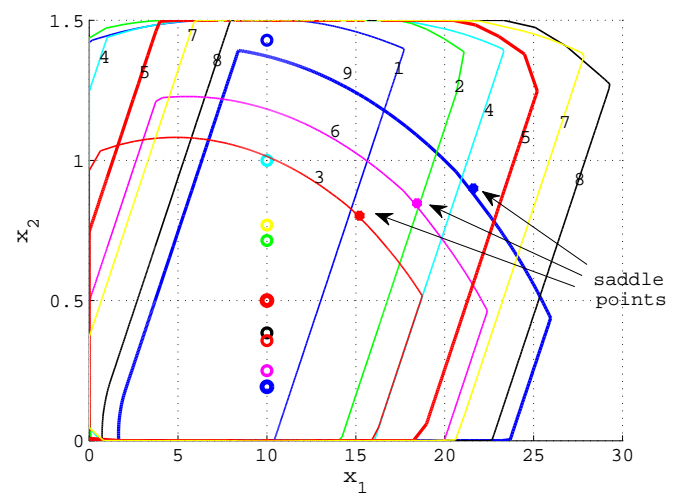

Figure 13: Family of contractive domains for various operating conditions $\left(V_{i n}=3.5,5,6.5 \mathrm{~V}, R=20,40,80 \Omega\right)$ for gain $\mathbf{k}_{2}=[0.0443-0.2324]^{T}$ and $V_{s s}=10 \mathrm{~V}$.

wide range of operating conditions needs to be addressed, so that further state and control constraints are satisfied and bifurcation phenomena are also ruled out, the proposed technique constitutes a simple and complete design method. All previous issues are taken a-priori into account and the designer is provided with enough degrees of freedom for desirable trade-offs between performance and safety.

\subsection{A different control gain choice}

To show the importance of the guidelines given by the diagram in Fig. 11, we consider a different control gain choice in the close neighborhood, but deliberately selected outside the feasible space. The new gain is

$$
\mathbf{k}_{2}=[0.0443,-0.2324]^{T}
$$

Both $\mathbf{k}_{1}, \mathbf{k}_{2}$ gains are marked with a "*" in Fig. 11 (b). It is obvious that the gain $\mathbf{k}_{2}$ is outside of the feasible subspace numbered 4 , hence it is expected to suffer from bifurcation phenomena. Next we carry out a full analysis to confirm this claim.

- For this gain complete bifurcation analysis using both the averaged and the switched model has been presented in

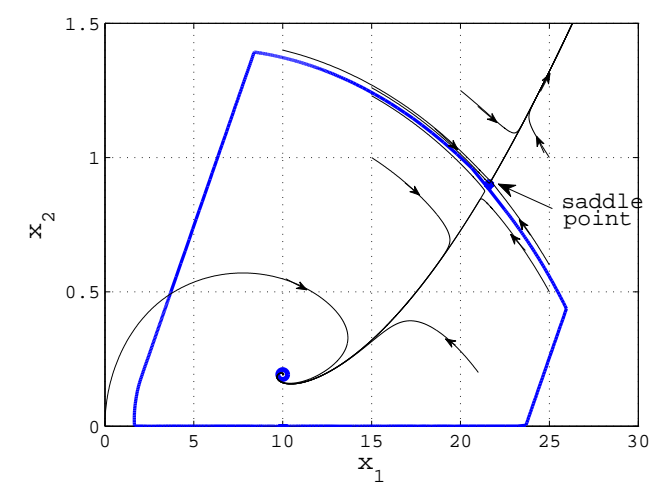

Figure 14: Multiple equilibria and several stable and unstable trajectories for gain $\mathbf{k}_{2}=[0.0443-0.2324]^{T}$ and $V_{s s}=10 \mathrm{~V}, V_{i n}=6.5 \mathrm{~V}, R=80 \Omega$.

section 3. The $V_{i n}-R$ bifurcation diagrams produced clearly suggest that, unlike the gain $\mathbf{k}_{1}$, serious multiple equilibria and period doubling phenomena appear inside the region of interest. This is also confirmed by the simulation results shown in subsection 2.3.

- Furthermore, in the presence of controller mismatch, Fig. 8 in subsection 3.1 reveals that, for gain $\mathbf{k}_{2}$, bifurcation phenomena are also present for relatively small variations of the uncertain parameters from their nominal values.

- Finally, a very illuminating picture for the effect of these phenomena is provided by the UDOAs shown in Fig. 13. By comparing Figs. 12,13, it is obvious that for high values of the output load $R$ the corresponding UDOAs are significantly reduced in size. It can be verified that this is due to the generation of multiple equilibria. In Fig. 13 an unstable attractor -a saddle point- appears, marked with a "*", being very close to the border of the UDOAs. Several trajectories inside and outside the UDOA region are shown in Fig. 14. The domain found is a very good estimate of the real UDOA. This is seen by observing that the diverging trajectories are very close to its boundary. In this case, three equilibria points are born, i.e. $(10,0.19)$-stable node,desired- , $(21.51,0.89)$ -saddle point- , $(67.61,8.79)$-stable node-.

Representative startup and load transient responses for nominal and extreme operating conditions for all gains have been already shown in subsection 2.3. These results show that, for the gain $\mathbf{k}_{1}$ designed in this section using the proposed methodology, a satisfactory response is obtained which is robust to disturbances due to large parameter variations in a wide operating region. The second gain $\mathbf{k}_{2}$ has been found to suffer from bifurcation phenomena in the case of large parameter variations. The same analysis can be similarly applied to the third gain $\mathbf{k}_{3}=[0.06,-0.19]^{T}$ considered in subsection 2.3 , as well. It can be shown that, although providing excellent results in nominal operating conditions, this design suffers from serious bifurcation phenomena even in the presence of relatively small disturbances, as evidenced by the simulation results presented in subsection 2.3 . 


\subsection{Simulation and experimental results}

In this section we present some further simulation results, in which a comparison of the performance between the proposed robust controller with gain $\mathbf{k}_{1}$ and a Lyapunov-based controller is attempted. To the best of our knowledge [15] is the only publication that offers a design procedure for controllers used in DC-DC converters that takes into account various bifurcation phenomena and therefore we compare our proposed method with the controller presented in [15].

More specifically, it has been recently shown [15] that with Lyapunov-based controllers (which use parameter-dependent gains, see (16)) multiple equilibria phenomena are avoided in the absence of a controller mismatch, while they appear far outside the range of feasible variations even in the case of a controller mismatch. Moreover, robust closed-loop stability is guaranteed, provided that input saturation is avoided. Hence, the design of a Lyapunov-based controller is reduced to the selection of a single parameter $\gamma$ such that saturation is avoided for the whole operating range, i.e. (54) is satisfied. For our example, the feasible interval found from (54) is $\gamma \leq 0.0283$. An optimal choice in that respect is to select the highest feasible value, i.e. $\gamma=0.0283$. This design is further validated with the systematic construction of a sufficiently dense family of UDOAs using polyhedral Lyapunov functions, whereby the converter's safe operation under state and control constraints for the whole operating regime has been confirmed.

The result of comparing this Lyapunov-based control design with the gain $\mathbf{k}_{1}$ proposed in the previous section is seen in Fig. 15. It is clear that a controller with two degrees of freedom can offer improved performance, since a better transient response with fewer oscillations (increased damping) and shorter settling time can be obtained. Hence, it is proven that the proposed methodology in this publication offers a satisfactory transient response while at the same time ensuring the avoidance of multiple equilibria and the presence of subharmonics that can greatly damage the converter.

Therefore, an important lesson learned by this research is that careful selection of the gain values is absolutely essential in order to avoid the presence of unstable attractors inside the region of interest, which will seriously affect the real region of stability. In this respect, an important contribution of the present paper is the proposal of useful bifurcation analysisbased criteria that can facilitate the design process.

Finally, the design is also experimentally verified using a prototype switched converter with the same components defined in section 5 and a hardware digital implementation of the controller using Labview on board the NI SBRIO 9636 FPGA device from National Instruments. The inductor current sensor was chosen to be a LEM LTS 6-NP. The evolution of the output voltage of the converter at start-up is shown in Fig. 15(c) together with the corresponding simulated response in order to verify their similarity.

Another interesting observation by the aforementioned results is that despite using a continuous-time model for the controller design, it was found that the effects of the digital implementation were negligible. This was confirmed by numerical

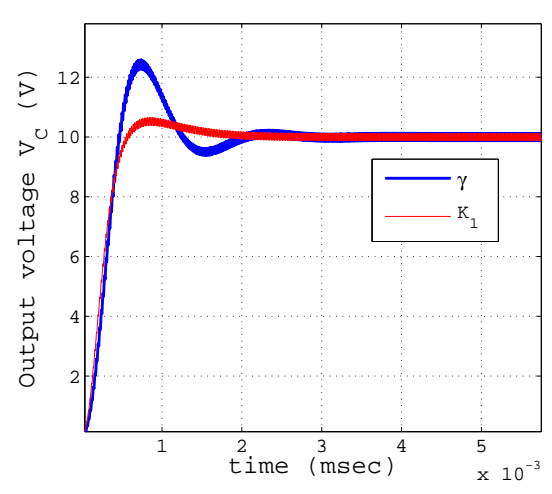

(a)

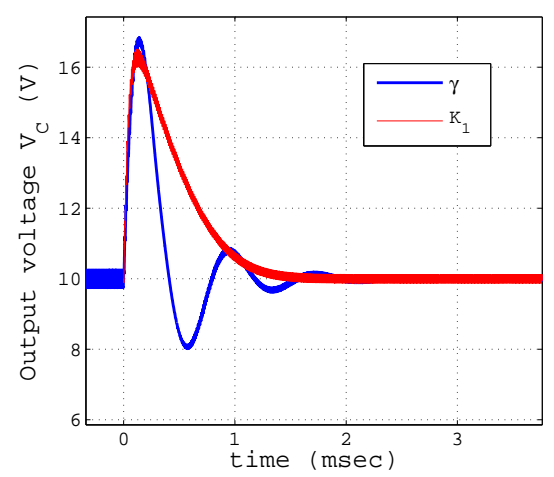

(b)

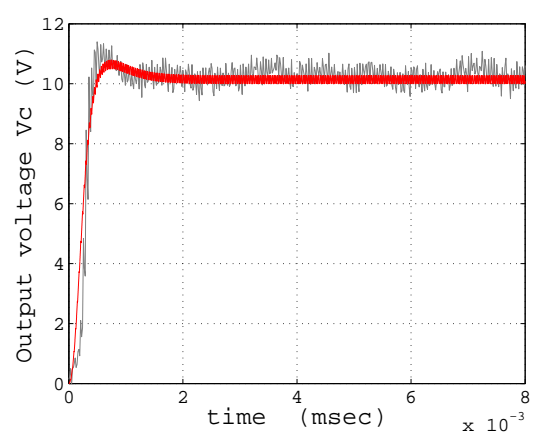

(c)

Figure 15: Comparison between gain $\mathbf{k}_{1}$ and Lyapunov-based controller $\gamma$ for $V_{s s}=10 \mathrm{~V}, V_{i n}=5 \mathrm{~V}, \gamma=0.0283$ for the output voltage. (a) Startup transient response with $R=40 \Omega$, (b) a load step change response for $R=$ $80 \rightarrow 20 \Omega$, (c) experimental startup waveform vs simulated response.

and experimental results and is due to the high sampling frequency compared to the converter's dynamics. More specifically, for the robust closed-loop system obtained with gain $\mathbf{k}_{1}$, for which the performance specifications $\zeta \geq 0.5, T_{s} \leq 2$ msec are guaranteed for the whole operating range, it can be shown using the linearized model (39) that the system's bandwidth $\omega_{B}$ satisfies $1068 \leq \omega_{B} \leq 2136 \mathrm{rad} / \mathrm{sec}$. This implies that, for sampling frequencies $f_{s}=10 \mathrm{KHz}$ or higher, we have $\omega_{s}>30 \times \omega_{B}$, which justifies the usual rule of thumb given in [25], hence we expect the digitally controlled system to behave close to its continuous counterpart. This is a common practice that can also be found in other publications, e.g. [11]. 


\section{Conclusion}

This paper presents a new method for the design of robust and efficient control laws for power converters under large parameter variations. The design is facilitated by specifying the feasible space of solutions in a non-conservative manner, as a result of imposing bifurcation analysis-based and other saturation avoidance and performance criteria. The constrained tracking problem is dealt with constrained stabilization ideas whereby the design is verified using the ray-gridding approach and the corresponding computer-generated set-induced PL Lyapunov functions.

The proposed design guarantees low complexity of the implementation, accurate nonlinear dynamics incorporation, nonconservative handling of hard state and control constraints, robustness to supply voltage variations and output load changes and satisfactory bifurcation behavior. We have shown that bifurcation analysis on the basis of the continuous-time averaged bilinear converter model provides faithful prediction of undesirable nonlinear phenomena, such as unstable attractors. It is an important ingredient of a complete control design process, in order to ensure the complete avoidance or the sufficient suppression of these phenomena.

Furthermore, although the results are reported for the case of a boost converter, we believe that the technique is applicable to other types of converters, as well. Future work will look at the problem of designing switching state-feedback control laws, that may offer larger domains of attraction or faster responses for wider operating regions. Further detailed comparison with more advanced techniques, such as hybrid MPC controllers, will be also investigated in future publications.

\section{APPENDIX}

PROOF OF LEMMA 1:

We consider the derivative of the two real solutions $R_{1,2}$ of (22) w.r.t. $V_{\text {in }}$

$$
\frac{d R_{1,2}}{d V_{i n}}=\frac{k_{2}}{V_{i n}^{2} k_{1}^{3}} \pm \frac{2 k_{2}}{k_{1}^{3 / 2} V_{s s}^{2}} V_{i n}
$$

The derivative is negative for any values s.t.

$$
V_{i n}<\left(\frac{V_{s s}^{2}}{2 k_{1}^{3 / 2}}\right)^{3 / 2}
$$

Moreover, if

$$
V_{i n}>\frac{k_{1} V_{s s}^{2}}{4 V_{s s}^{2} k_{1}^{4}-1}
$$

both real solutions satisfy $R_{1,2}>0$. This proves that, if (59) and (60) are satisfied, there exist two separate monotonically decreasing curves as $V_{i n}$ increases for any values of $V_{s s}, k_{1}, k_{2}$. The two curves do not intersect, since there are always two different solutions $R_{1} \neq R_{2}$. Hence, for a-priori known bounds for the varying parameters $V_{i n}, R$ we are able to specify precisely the bifurcation boundary between one and three real equilibria. There are always three different regions, the area $\mathcal{A}_{1}$ above the top curve (three equilibria), the area $\mathcal{A}_{2}$ between the two curves (one equilibrium), and the area $\mathcal{A}_{3}$ below the bottom curve (three equilibria). Moreover, since only positive values of $V_{i n}, R$ are meaningful, these properties refer to the first quadrant of the $V_{i n}-R$ plane.

\section{PROOF OF LEMMA 2:}

For fixed $V_{i n}, R$, the bifurcation curve can also be seen as a parabola on the $k_{1}-k_{2}$ plane, since the corresponding discriminant vanishes, i.e. $B^{2}-4 A C=0$, where $A=V_{s s} V_{i n}^{2} R^{2}, B=$ $2 V_{\text {in }} R V_{s s}^{2}, C=V_{s s}^{3}$. On the $k_{1}-k_{2}$ plane, in the fourth quadrant of interest $k_{1}>0, k_{2}<0$, there are always two separate monotonically decreasing curves. From the definition of $\Gamma$, we derive for $k_{1}=0$ two solutions $k_{2}=0, k_{2}=-\frac{4 R V_{i n}^{2}}{V_{s s}^{3}}<0$, while for fixed larger positive values $k_{1}>0$ we get a quadratic in $k_{2}$

$$
k_{2}^{2}+\frac{2 V_{i n} R V_{s s}^{2} k_{1}+4 R V_{i n}^{2}}{V_{s s}^{3}} k_{2}+\frac{V_{s s} V_{i n}^{2} R^{2} k_{1}^{2}}{V_{s s}^{3}}=0
$$

which gives us always two solutions since its discriminant is given by

$$
\Delta_{3}=\frac{16 R^{2} V_{i n}^{3}\left(V_{i n}+k_{1} V_{s s}^{2}\right)}{V_{s s}^{6}}
$$

and it is always positive for any values of $k_{1}>0$.

The two solutions are

$$
k_{21,22}=\frac{-V_{i n} R V_{s s}^{2} k_{1}-2 R V_{i n}^{2} \pm 2 R V_{i n}^{\frac{3}{2}} \sqrt{V_{i n}+k_{1} V_{s s}^{2}}}{V_{s s}^{3}}
$$

Taking the derivative of $k_{21,22}$ w.r.t. $k_{1}$ gives

$$
\frac{d k_{21,22}}{d k_{1}}=\frac{-V_{i n}^{3 / 2} R\left(\sqrt{1+\frac{k_{1} V_{s s}^{2}}{V_{i n}}} \pm 1\right)}{V_{s s} \sqrt{V_{i n}+k_{1} V_{s s}^{2}}}<0, \forall k_{1}>0
$$

This proves the existence of two separate monotonically decreasing curves as $k_{1}$ increases for any values of $V_{s s}, V_{i n}, R$. The first curve starts from the origin and the second one from a lower negative value $k_{2}=-\frac{4 R V_{i n}^{2}}{V_{s s}^{3}}$. The two curves do not intersect, since there are always two different solutions $k_{21,22}$. All these suggest, that for a-priori known bounds for the varying parameters $V_{i n}, R$ we are able to specify precisely the bifurcation boundary between one and three real equilibria. Again, we are always going to have three different regions, the area $\mathcal{B}_{1}$ above the top curve (three equilibria), the area $\mathcal{B}_{2}$ between the curves (one equilibrium), and the area $\mathcal{B}_{3}$ below the bottom curve (three equilibria). Furthermore, since only values satisfying $k_{1}>0, k_{2}<0$ are studied, these properties refer to the fourth quadrant of the $k_{1}-k_{2}$ plane.

\section{PROOF OF LEMMA 3:}

A simple formula for the locus of the controller gains that ensure the absence of positive real equilibria can be found from (19). The two new equilibria that are born when the bifurcation curve is crossed are

$$
v_{1,2}=-\frac{1}{2}\left(p_{1} \pm \sqrt{p_{1}^{2}-4 q_{1}}\right), q_{1}>0
$$


From this expression it is clearly seen that if $p_{1}>0$ then both solutions satisfy $v_{1,2}<0$, hence the converter's stability is not affected. On the other hand, if $p_{1}<0$ both solutions are positive, hence their position may affect the stability of the converter's system. The condition $p_{1}>0$ implies $-\frac{k_{1}}{k_{2}}<\frac{V_{s s}}{R V_{i n}}$. For uncertainty intervals as in (11) this holds for any values of the uncertain parameters $V_{i n}, R$ if and only if (25) is satisfied. The lemma is proved.

\section{PROOF OF LEMMA 4:}

We impose the condition $v_{1,2}>V_{C}^{+}$. In this case we have $p_{1}<0$ and it is enough to ensure that the smallest of the two roots satisfies the condition. From (65) we get

$$
-\frac{1}{2}\left(p_{1}+\sqrt{p_{1}^{2}-4 q_{1}}\right)>V_{C}^{+} \Rightarrow p_{1}>-V_{C}^{+}-q_{1} / V_{C}^{+}
$$

Further manipulations lead to (25) and the lemma is proved.

\section{References}

[1] M. di Bernardo, C. Budd, A. R. Champneys, P. Kowalczyk, Piecewisesmooth Dynamical Systems, Springer-Verlag, London, 2008.

[2] C. K. Tse, Complex Behavior of Switching Power Converters, CRC Press, Boca Raton, USA, 2003.

[3] S. Banerjee, G. C. Verghese (Eds.), Nonlinear Phenomena in Power Electronics: Attractors, Bifurcations, Chaos, and Nonlinear Control, IEEE Press, New York, 2001.

[4] S. Mariethoz, et al, Comparison of hybrid control techniques for buck and boost DC-DC converters, IEEE Transactions on Control Systems Technology 18 (5) (2010) 1126-1145.

[5] A. Soldatos, P. Karamanakos, K. Pavlou, S. Manias, Nonlinear robust control for DC-DC converters, in: ICECS, 2010, pp. 994-997.

[6] V. Spinu, N. Athanasopoulos, M. Lazar, G. Bitsoris, Stabilization of bilinear power converters by affine state feedback under input and state constraints, IEEE Transactions on Circuits and Systems-II Express Briefs 59 (8) (2012) 520-524.

[7] D. Plaza, R. D. Keyser, J. Bonilla, Model predictive and sliding mode control of a boost converter, in: SPEEDAM, 2008, pp. 37-42.

[8] S. Almer, S.Mariethoz, M. Morari, Piecewise Affine Modeling and Control of a Step-up DC-DC converter, in: American Control Conference, 2010, pp. 3299-3304.

[9] V. Spinu, M. Lazar, Integration of real-time and stability constraints via hybrid polytopic partitions, in: IEEE CCA, 2012, pp. 226-233.

[10] T. Hu, A nonlinear-system approach to analysis and design of powerelectronic converters with saturation and bilinear terms, IEEE Transactions on Power Electronics 26 (2011) 399-410.

[11] C. Olalla, R. Leyva, I. Queinnec, D. Maksimovic, Robust Gain-Scheduled Control of Switched-mode DC-DC Converters, IEEE Transactions on Power Electronics 27 (2012) 3006-3019.

[12] C. Olalla, I. Queinnec, R. Leyva, A. E. Aroudi, Optimal state-feedback control of bilinear DC-DC Converters with guaranteed regions of stability, IEEE Transactions on Industrial Electronics 59 (2012) 3868-3880.

[13] V. Spinu, M. Lazar, P. van den Bosch, An explicit state-feedback solution to constrained stabilization of DC-DC power converters, in: IEEE CCA, 2011, pp. 1112-1118.

[14] C. Yfoulis, D. Giaouris, S. Voutetakis, S. Papadopoulou, Constrained switching stabilization of a dc-dc boost converter using piecewise-linear Lyapunov functions, in: IEEE MED, 2013, pp. 814-823.

[15] M. Spinetti-Rivera, J. Olm, D. Biel, E. Fossas, Bifurcation analysis of a Lyapunov-based controlled boost converter, Communications in Nonlinear Science and Numerical Simulation 18 (2013) 3108-3125.

[16] D. Giaouris, C. Yfoulis, S. Voutetakis, S. Papadopoulou, Stability analysis of state feedback controlled boost converters, in: Proceedings of the IECON 2013 - 39th Annual Conference of the IEEE Industrial Electronics Society, 2013, pp. 8383-8388.

[17] C. Yfoulis, D. Giaouris, F. Stergiopoulos, C. Ziogou, S. Voutetakis, S. Papadopoulou, Flexible polyhedral Lyapunov functions for the robust con- strained stabilization of bilinear boost DC-DC converters, in: IEEE MED, 2014, pp. 1261-1266.

[18] R. Leyva, L. Salamero, H. Valderamma-Blavi, Linear State-Feedback Control of a Boost Converter for Large-Signal Stability, IEEE Transactions on Circuits and Systems-I 48 (2001) 418-424.

[19] R. Keyser, J. Bonilla, C. Ionescu, A comparative study of several control techniques applied to a boost converter, in: IEEE 10th Int Conf on Optimisation of electrical and electronic equipment OPTIM, 2006, pp. 71-78.

[20] F. M. Oettmeier, J. Neely, S. Pekarek, R. DeCarlo, K. Uthaichana, MPC of Switching in a Boost Converter Using a Hybrid State Model With a Sliding Mode Observer, IEEE Transactions on Industrial Electronics 56 (2009) 3453-3466.

[21] C. Yfoulis, A. Muir, P. Wellstead, A new approach for estimating controllable and recoverable regions for systems with state and control constraints, Int. Journal of Robust and Nonlinear Control 12 (2002) 561-589.

[22] C. A. Yfoulis, R. Shorten, A numerical technique for the stability analysis of linear switched systems, Int. Journal of Control 77 (2004) 1019-1039.

[23] C. A. Yfoulis, Constrained switching stabilization of linear uncertain switched systems using piecewise linear lyapunov functions, Transactions of the Institute of Measurement and Control 32(5) (2010) 529-566.

[24] N. Athanassopoulos, M. Lazar, G. Bitsoris, On the construction of invariant proper C-polytopic sets for continuous-time linear systems, in: ICSTCC, 2012, pp. 1-6.

[25] G. Franklin, J. Powell, M. Workman, Digital control of dynamic systems, Addison-Wesley, 3rd edition, 1997. 